\title{
Variational Integrators for Dissipative Systems
}

\author{
David J. N. Limebeer, IEEE Life Fellow, Sina Ober-Blöbaum and Farhang Haddad Farshi
}

\begin{abstract}
This paper uses physical arguments to derive variational integration schemes for dissipative mechanical systems. These integration algorithms find utility in the solution of the equations of motion and optimal control problems for these systems. Engineers usually represent dissipation effects using phenomenological devices such as 'dampers'. In the work presented here we replace these dampers with a lossless transmissionline in order that the equations of motion are derivable from a variational principle. The associated system Lagrangian can then be discretized and used to develop low-order variational integration schemes that inherit the advantageous features of their conservative counterparts. The properties of a lossless spring-inerter based transmission system are analyzed in detail, with the resulting variational integration schemes shown to have excellent numerical properties. The paper concludes with the analysis of a dissipative variant of the classical Kepler central force problem.
\end{abstract}

\section{INTRODUCTION}

$\mathbf{N}$ Umerical methods for solving optimal control problems rely on discretizing the underlying (continuous-time) dynamical system equations. This (transcription) process transforms the problem into a nonlinear programming problem (NLP) (see e.g. [1]). While this procedure is well established in general terms, there are still difficulties that limit the scope and utility of this approach in the case of (electro-)mechanical systems: (i) mechanical modelling and optimal control are based on closely related variational principles, yet this common heritage is not exploited by most standard solution processes; (ii) general purpose numerical integration algorithms can destroy the geometric structure of the underlying system model. In the context of conservative systems inappropriate integration schemes may produce numerical dissipation/energy injection, and can destroy other conserved quantities such as the system's linear and/or angular momentum [2]-[4]. (iii) Since the special structure of (electro-)mechanical models is not exploited in the standard approach, the number of decision variables is needlessly doubled. This follows from the unnecessary retention of the generalized velocities in the system's state vector. In discrete mechanical models (models based on a discrete Euler-Lagrange equation) the generalized velocities are expressible in terms of time differences in the generalized positions and thus can be eliminated from the problem. Some of these ideas have been developed in the context of astrodynamics and dynamical astronomy, and have been applied to the determination of the motion of objects in space [5], [6].

David J. N. Limebeer (david.limebeer@eng.ox.ac.uk) is a Distinguished Professor at the University of Johannesburg, South Africa. Sina OberBlöbaum (sina.ober-blobaum@eng.ox.ac.uk) and Farhang Haddad Farshi (farhang.haddadfarshi@eng.ox.ac.uk) are with the Department of Engineering Science, University of Oxford, Parks Road, Oxford, OX1 3PJ, phone: +44 1865 273153. This work has been part funded by the EPSRC project: "Fractional Variational Integration and Optimal Control"; ref: EP/P020402/1.
Variational integrators for conservative systems are derived by discretizing the Hamiltonian/Lagrangian prior to deriving (discrete) Euler-Lagrange equations [7]. To see how this process works, consider the simple approximation of a Lagrangian action on the time interval $[0, T]$ using a forward difference operator to approximate the velocity $\dot{q}$, and a left-end point for the position so that

$$
\begin{aligned}
\int_{0}^{T} \mathcal{L}(q(t), \dot{q}(t)) d t & \approx \sum_{k=0}^{N-1} h \mathcal{L}\left(q_{k}, \frac{q_{k+1}-q_{k}}{h}\right) \\
& \approx \sum_{k=0}^{N-1} \mathcal{L}_{d}\left(q_{k}, q_{k+1}, h\right)
\end{aligned}
$$

with $q_{k} \approx q(k h)$, and $q_{k+1} \approx q((k+1) h)$ and time step $h$ with $N h=T$. Taking discrete variations, using discrete summation by parts, yields the discrete Euler-Lagrange equations

$$
\begin{aligned}
D_{1} \mathcal{L}_{d}\left(q_{k}, q_{k+1}, h\right) & +D_{2} \mathcal{L}_{d}\left(q_{k-1}, q_{k}, h\right)=0, \\
\text { for } \quad k & =1, \ldots, N-1,
\end{aligned}
$$

where $D_{i}$ denotes the partial derivative with respect to the $i$-th argument. The solution of (1) constitutes a discrete flow map of the variational integrator-the approximation given in (1) results in a variational Euler integration scheme. This process yields directly unified numerical schemes with powerful structure-preserving properties. These variational ${ }^{1}$ integrators satisfy a variational principle [7], [8], and the geometric characteristics of the underlying continuous system are inherited by the discrete approximation. Variational integrators have been shown to have excellent long-term energy preserving properties, which stems from the fact that the (discrete) flows correspond to the exact flows of a neighbouring discrete Lagrangian ( [9]).

One way to incorporate a non-conservative force term $F$ in the Lagrangian formulation is based on the Lagranged'Alembert principle (e.g. [10], [11]). Instead of considering the stationarity of the action, the variation of the action is set equal to the work $\int F \cdot \delta q d t$ done by the external forces when the system undergoes an arbitrary virtual displacement. This results in ${ }^{2}$

$$
d_{t}\left(\partial_{\dot{q}} L\right)-\partial_{q} L=F,
$$

which is no longer based on a variational principle. In some contexts it may be useful to set $F=\partial_{\dot{q}} \varphi(q, \dot{q})$, where $\varphi(q, \dot{q})$ is a dissipation potential [11]. The forced discrete Euler-

\footnotetext{
${ }^{1}$ In the case of conservative systems any variational integrator is also symplectic, because it preserves the system's symplectic geometry.

${ }^{2}$ The $n^{t h}$ full and partial derivatives with respect to the subscripted argument argument are given by $d^{n}$ and $\partial^{n}$ respectively. We will use $\dot{q}$ and $\ddot{q}$ to represent first- and second-order derivatives with respect to time.
} 
Lagrange equations are given by

$$
\begin{array}{rcc}
D_{1} \mathcal{L}_{d}\left(q_{k}, q_{k+1}, h\right)+D_{2} \mathcal{L}_{d}\left(q_{k-1}, q_{k}, h\right) & + & \\
F_{d}^{+}\left(q_{k-1}, q_{k}\right)+F_{d}^{-}\left(q_{k}, q_{k+1}\right) & = & 0 \\
\text { for } k=1, \ldots, & N-1 . &
\end{array}
$$

If we choose the approximation $F_{d}^{-}\left(q_{k}, q_{k+1}\right)=0$ and $F_{d}^{+}\left(q_{k}, q_{k+1}\right)=h F\left(q_{k}, \frac{q_{k+1}-q_{k}}{h}\right)$ for a dissipative force $F=-\Gamma \dot{q}$, the dissipation is approximated by the backward difference operator. Variational integrators for dissipative systems, which are based on the Lagrange-d'Alembert principle, have been described in [8], [11]-[14]. The resulting integrators show excellent energy decay rate tracking properties in specific examples, but there are no general proven results explaining this phenomenon.

Extensions to constrained ( [15]-[18]), non smooth ( [19]), stochastic ( [20]), multi-rate and multi-scale ( [21]-[23]), Lagrangian PDE systems ( [24]-[26]), electric circuits ( [27]) and optimal control problems ( [13], [28]) show consistently superior qualitative behaviour when compared to classical methods.

The challenge of synthesizing variational integrators for dissipative systems has a fascinating counterpart in physics: 'how does one describe irreversible (dissipative) behaviours using Hamiltons principle?', which is a question of central importance in quantum mechanics [29]. In accepted theories of dissipation, damping is a collective phenomenon involving the interaction of a master particle with a background constellation of secondary particles known collectively as a heat bath. The dissipation of energy to the heat bath causes damping. At the same time, the master particle receives energy from fluctuations in the heat bath - this is the subject of fluctuation dissipation theory [30]. The excellent report [29] provides a comprehensive review of dissipation phenomena in Lagrangian and Hamiltonian mechanics.

For conservative systems variational methods are equivalent to Newton's laws. However, while Newton's equations accommodate non-conservative forces, variational techniques have no way to dealing with them straightforwardly. In quantum mechanics there have been a number of attempts to describe dissipation mechanisms within variational frameworks [29]. The importance of variational methods can be summed up as follows [31]: '... most physicists would be not only willing to accept as axiomatic the existence of a variational principle, but would be loath to accept any dynamic equations that were not derivable from such a principle ...'

Section II outlines a number of approaches to the modelling of dissipation mechanisms using variational principles. While this material is of great interest, and may well act as the basis for other approaches to the design of variational integrators, it need not be studied in detail in the first instance. Section III examines the use of a transmission-line-based heat bath as part of a closed conservative system that enables variational integration schemes to be derived. We will use a (lossless) transmission line that is made up of springs and inerters [32]; in special cases the inerters can be replaced with masses. Section III develops the describing equations of a closed dualoscillator-transmission-line system and shows that it is equiva- lent to an open system that includes a linear phenomenological damper for an arbitrarily long time interval. Importantly, all the relevant equations are derivable from variational principles. Section IV shows that the results in Section III can be extended to the discrete-time case. These discrete-time results can be used to derive discrete-time variational integration schemes for the open dual-oscillator system. These results are used to solve a dissipative central force problem in Section V, where a variational integration scheme is developed for a dissipative variant of the Kepler problem. The superior performance of the variational integration scheme, as compared with nonvariational counterparts, is thus demonstrated. The conclusions appear in Section VI.

\section{CAndidate Approaches}

Variational principles are ubiquitous in theoretical physics. Our purpose here is to review a number of possible approaches to the use of variational methods for modelling dissipative systems.

An example of an electric circuit law being determined by a variational principle is due to Kirchhoff [33]; this law was apparently also known to Maxwell (see paragraph 284, pp 337, [34]). Consider a region of space $V$ with conductivity $\sigma(\mathbf{r})$ at location $\mathbf{r}$. Suppose that the electric field in $V$ is given by $\mathbf{E}(\mathbf{r})=-\nabla \phi(\mathbf{r})$, where $\phi(\mathbf{r})$ is the electric field potential. The power dissipated in $V$ is given by

$$
\mathcal{P}=\int_{V} \sigma(\nabla \phi)^{2} d V
$$

If the potential is known and fixed on the boundary of $V$

$$
\delta \mathcal{P}=0 \quad \Leftrightarrow \quad \sigma \nabla \cdot(\nabla \phi)=0 ;
$$

which requires that there is no charge accumulation in $V$. Equation (3) is the Euler-Lagrange equation associated with the action (2), and is a field-theoretic statement of Kirchhoff's current law. If Kirchhoff's current law holds, the current distributes itself in a way that minimizes the total heat dissipation in $V$ (for any given potential distribution on the boundary).

Cherry [35] considers the case of circuits containing nonlinear resistors, nonlinear inductors and nonlinear capacitors. One can associate the circuit energy $U$ with the capacitors, the circuit co-energy $T$ with the inductors and the circuit content $G$ with the circuit resistors ${ }^{3}$. If the circuit mesh currents are given by $\dot{q}_{1}, \dot{q}_{2}, \cdots, \dot{q}_{n}$, and the circuit Lagrangian is given by $\mathcal{L}=T(\dot{\boldsymbol{q}})-U(\boldsymbol{q})$, then

$$
d_{t}\left(\partial_{\dot{q}_{r}} \mathcal{L}\right)-\partial_{q_{r}} \mathcal{L}=-\partial_{\dot{q}_{r}} G \quad r=1,2, \cdots, n
$$

gives the equations for the mesh currents. The dissipative potential term $\partial_{\dot{q}_{r}} G$ means that (4) are not Euler-Lagrange equations of the type associated with a variational principle. The resistive term is reminiscent of a Rayleigh dissipation term of the type used to describe damping forces proportional to velocity; see [36] pages 24-24, and [11].

\footnotetext{
${ }^{3}$ Co-energy and content are defined in [35].
} 
Another attempt to describe dissipation in a variational framework is the Kanai-Caldirola (KC) oscillator [37], [38]. In this case the Lagrangian takes the form

$$
\mathcal{L}=\frac{e^{\Gamma t}}{2}\left(\dot{x}^{2}-\omega^{2} x^{2}\right)
$$

It follows from Lagrange's equation that

$$
d_{t}\left(\partial_{\dot{x}} \mathcal{L}\right)-\partial_{x} \mathcal{L}=e^{\Gamma t}\left(\ddot{x}+\Gamma \dot{x}+\omega^{2} x\right)=0 .
$$

In one interpretation of (6), the mass is thought of as timevarying, and the conjugate momentum $p=e^{\Gamma t} \dot{x}$ makes physical sense as the momentum of an accreting mass-in this interpretation no dissipation takes place [39]. In another interpretation, the $e^{\Gamma t}$ term is simply 'ignored', and (5) is the Lagrangian of a dissipative system. In this interpretation the conjugate momentum and Hamiltonian are difficult to interpret physically.

In yet another attempt to find a variational description of dissipation, Bateman, Morse, Feshbach, and Tikochinsky (BMFT) [40]-[42] developed an oscillator described by the Lagrangian

$$
\mathcal{L}=\dot{x} \dot{y}+\frac{\Gamma}{2}(x \dot{y}-y \dot{x})-\omega^{2} x y .
$$

It follows from Langrange's equations that

$$
d_{t}\left(\partial_{\dot{y}} \mathcal{L}\right)-\partial_{y} \mathcal{L}=\ddot{x}+\Gamma \dot{x}+\omega^{2} x=0
$$

and

$$
d_{t}\left(\partial_{\dot{x}} \mathcal{L}\right)-\partial_{x} \mathcal{L}=\ddot{y}-\Gamma \dot{y}+\omega^{2} y=0 .
$$

The first equation describes the motion of a damped mass, while the second describes the motion of an anti-damped mass. In this case the conjugate momenta are given by $p_{x}=\dot{y}-\frac{\Gamma y}{2}$ and $p_{y}=\dot{x}+\frac{\Gamma x}{2}$, which again, have no clear physical meaning.

It is argued in [43] that the $\mathrm{KC}$ oscillator described by (5) and the BMFT oscillator described by (7) are equivalent, lead to the same Euler-Lagrange equations, and describe the same physical system. To see this we begin with (7) and exploit the fact that the Euler-Lagrange equations resulting from ${ }^{4}$

$$
\mathcal{L}_{0}=\mathcal{L}-d_{t} f=\dot{x} \dot{y}+\frac{\Gamma}{2}(x \dot{y}-y \dot{x})-\omega^{2} x y-d_{t} f
$$

in which

$$
f=\frac{\Gamma}{4}\left(x^{2} e^{\Gamma t}+y^{2} e^{-\Gamma t}\right)
$$

are the same as those resulting from (5).

Another approach to the variational theory of dissipation is to modify Hamilton's principle itself rather than 'invent' novel Lagrangians. One such re-formulation is given in [44], where the 'doubling of the state dimension' theme recurs. The classical Lagrangian $\mathcal{L}(q, \dot{q})$, is replaced by $\mathcal{L}\left(q_{1}, \dot{q}_{1}\right)$, $\mathcal{L}\left(q_{2}, \dot{q}_{2}\right)$, and a generalized potential $\mathcal{K}\left(q_{1}, q_{2}, \dot{q}_{1}, \dot{q}_{2}\right)$ that plays the key role in producing nonconservative forces. The

$$
\begin{aligned}
& { }^{4} \text { Suppose } \mathcal{L}_{0}=\mathcal{L}-d_{t} f, \text { then } \\
& \qquad \mathcal{S}_{0}=\int \mathcal{L}_{0} d t=\int \mathcal{L}-f_{B}+f_{A} .
\end{aligned}
$$

The additional terms are fixed and have no influence on the equations of motion. associated action is

$\mathcal{S}_{G}=\int_{t_{i}}^{t_{f}}\left(\mathcal{L}\left(q_{1}, \dot{q}_{1}\right)+\mathcal{K}\left(q_{1}, q_{2}, \dot{q}_{1}, \dot{q}_{2}\right)\right) d t+\int_{t_{f}}^{t_{i}} \mathcal{L}\left(q_{2}, \dot{q}_{2}\right) d t$

in which the generalized potential is given by $K\left(q_{1}, q_{2}, \dot{q}_{1}, \dot{q}_{2}\right)=\frac{1}{2} F\left(q_{2}, \dot{q}_{2}\right)\left(q_{1}-q_{2}\right)-\frac{1}{2} F\left(q_{1}, \dot{q}_{1}\right)\left(q_{2}-q_{1}\right)$ [45]. The boundary conditions associated with (8) are: (1) $q_{1}\left(t_{i}\right)$ and $q_{2}\left(t_{i}\right)$ are fixed, (2) $q_{1}\left(t_{f}, \epsilon\right)=q_{2}\left(t_{f}, \epsilon\right)$ and (3) $\dot{q}_{1}\left(t_{f}, \epsilon\right)=\dot{q}_{2}\left(t_{f}, \epsilon\right)$ for all variations $\epsilon$. Conditions (2) and (3) do not fix the final state as in the classical boundary-value problem formulation, they simply demand that they are equal. The forward- and reverse-time line integrals and the boundary conditions associated with the action (8) are illustrated in Figure 1. As demonstrated in [44], stationarity of the action

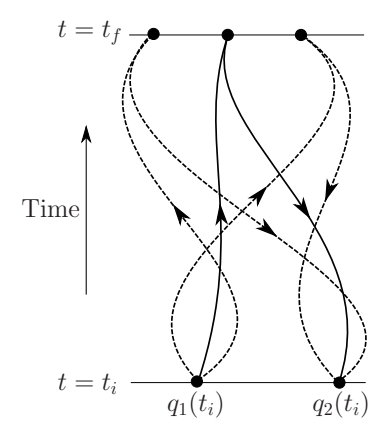

Fig. 1: Re-formulation of Hamilton's principle. The solid lines denote the stationary path, while the dotted lines denote virtual displacements. The arrows indicate the integration direction for the line integrals in the action.

results in a pair of Euler-Lagrange equations

$$
\frac{d}{d t}\left(\frac{\partial \mathcal{L}}{\partial \dot{q}_{1}}\right)-\frac{\partial \mathcal{L}}{\partial q_{1}}=0 \quad \frac{d}{d t}\left(\frac{\partial \mathcal{L}}{\partial \dot{q}_{2}}\right)-\frac{\partial \mathcal{L}}{\partial q_{2}}=0 .
$$

Once equations (9) have been evaluated, the equations of motion are recovered by taking the physical limit $q_{2} \rightarrow q_{1}=q$ and $\dot{q}_{2} \rightarrow \dot{q}_{1}=\dot{q}^{5}$

It is shown in [46] that the equations of motion of linear dissipative system with constant coefficients cannot be derived using a variational principle. While this appears initially to be a discouraging message, Bauer left the door open for systems containing time-varying masses, embedding the system in various types of heat bath, and the use of non-integer calculus. Approaches based on fractional calculus stem from the following simple observation [47], [48]: if the Lagrangian/Hamiltonian contains a term proportional to $\left(d_{t}^{n}\right)^{2}$, then the Euler-Lagrange equation will have a corresponding term proportional to $d_{t}^{2 n}$. Therefore a damping force of the form $\Gamma \dot{x}$ should follow from a Lagrangian containing a term proportional to the fractional derivative $\left(d_{t}^{1 / 2}\right)^{2}$. Riewe derived a Euler-Lagrange equation for functionals dependent on left and right Riemann-Liouville derivatives $\mathcal{D}_{-}^{\alpha}$ and $\mathcal{D}_{+}^{\alpha}$ for $\alpha>0$. If one considers a

\footnotetext{
${ }^{5}$ This is the limiting process that produces the 'correct physics' and causes the equations in (9) to collapse into the nonconservative equations of motion [44].
} 
Lagrangian of the form

$$
\mathcal{L}\left(\dot{x}, x_{+}, x\right)=\frac{1}{2} \dot{x}^{2}+\frac{1}{2} \Gamma\left(\mathcal{D}_{+}^{1 / 2} x\right)^{2}-U(x),
$$

Riewe obtained

$$
\ddot{x}+\Gamma \mathcal{D}_{-}^{1 / 2} \mathcal{D}_{+}^{1 / 2} x+\partial_{x} U=0 .
$$

However

$$
\mathcal{D}_{-}^{1 / 2} \mathcal{D}_{+}^{1 / 2} \neq d_{t}
$$

and so Riewe's derivation of a variational principle for the linear damping problem required repair [49]. In a corrected treatment of the problem a doubling of the state-space dimension again results, and an anti-stable mirror system is again required:

$$
\begin{aligned}
\ddot{x}+\Gamma \dot{x}+\omega^{2} x & =0 \\
\ddot{y}-\Gamma \dot{y}+\omega^{2} y & =0 .
\end{aligned}
$$

As before, the fractional derivative treatment in [49], [50] introduces an anti-stable oscillator, which is the situation we found earlier for the BMFK and CK oscillators.

The quantum mechanics literature's point of closest approach to the work presented in this paper is the CaldeiraLeggett system-plus-reservoir model used to study Brownian motion [51]-[53].

\section{A. Caldeira-Leggett model}

Quantum mechanics analysis is usually conducted within a Hamiltonian framework, where the total energy of the system is a conserved quantity. It is not possible to describe dissipative systems this way. To overcome this difficulty the system under study is embedded in a heat bath, so that the combination of system and heat bath is conservative. To ensure an irreversible flow of energy (i.e., avoid Poincare recurrences), the bath requires (ideally) an infinite number of degrees of freedom.

1) Closed system Lagrangian: The closed system comprises a one-degree-of-freedom oscillator (the system $S$ ), a heat bath $(B)$ described by a set of $n$ harmonic oscillators, and bilinear coupling dynamics $(S B)$. The Lagrangian for the full system is given by

$\mathcal{L}=\overbrace{\frac{\dot{q}^{2}}{2}-\frac{\Omega_{0}^{2}}{2} q^{2}}^{\mathcal{L}_{S}}+\overbrace{\sum_{i=1}^{n}\left(\frac{m_{i}}{2} \dot{q}_{i}^{2}-\frac{k_{i}}{2} q_{i}^{2}\right)}^{\mathcal{L}_{B}}+\overbrace{\sum_{i=1}^{n} \frac{k_{i}}{2} q\left(2 q_{i}-q\right)}^{\mathcal{L}_{S B}}$.

The system Lagrangian is $\mathcal{L}_{S}$, the heat bath Lagrangian is $\mathcal{L}_{B}$, and the coupling Lagrangian is $\mathcal{L}_{S B}$.

2) Heat bath elimination: Solving Lagrange's equations gives

$$
\begin{aligned}
\ddot{q}+\Omega_{0}^{2} q+\sum_{i=1}^{n} k_{i} q & =\sum_{i=1}^{n} k_{i} q_{i} \\
\ddot{q}_{i}+\omega_{i}^{2} q_{i} & =k_{i} q \quad i=1, \cdots, n,
\end{aligned}
$$

where $\omega_{i}^{2}=k_{i} / m_{i}$. Supposing that $q(t)$ is a given function, equations (11) can be solved as

$$
\begin{aligned}
q_{i}(t)= & q_{i}(0) \cos \left(\omega_{i} t\right)+\frac{\dot{q}_{i}(0)}{\omega_{i}} \sin \left(\omega_{i} t\right) \\
& +\int_{0}^{t} d s \omega_{i} \sin \left(\omega_{i}(t-s)\right) q(s),
\end{aligned}
$$

where $q_{i}(0)$ and $\dot{q}_{i}(0)$ are initial conditions. Substituting (12) into (10) and integrating by parts gives

$$
\ddot{q}+\Omega_{0}^{2} q+\int_{0}^{t} d s\left(\sum_{i=1}^{n} k_{i} \cos \left(\omega_{i}(t-s)\right)\right) \dot{q}(s)=F(t),
$$

where

$F(t)=\sum_{i=1}^{n} k_{i}\left(\left(q_{i}(0)-k_{i} q(0)\right) \cos \left(\omega_{i} t\right)+\frac{\dot{q}_{i}(0)}{\omega_{i}} \sin \left(\omega_{i} t\right)\right)$,

which represents a zero-mean random force that depends on the initial conditions of the system and bath. The third term on the left-hand side of (13) is a damping term. In the limit $\lim _{n \rightarrow \infty}$, if $k_{i}=2 \Gamma$ and the $\omega_{i}$ are 'evenly spaced',

$$
\sum_{i=1}^{n} k_{i} \cos \left(\omega_{i}(t-s)\right)=2 \Gamma \delta(t-s)
$$

and constant linear (Ohmic) damping is achieved [54]. Since the integral in (13) ends at $s=t$, the delta function only counts half, and we obtain

$$
\ddot{q}+\Gamma \dot{q}+\Omega_{0}^{2} q=F .
$$

Equation (14) is the Langevin equation [30], [52], and describes a noise-driven system with damping-although the (closed) system is conservative. In problems of this type highorder closed conservative descriptions can be replaced by loworder models with linear 'phenomenological' dissipation. This behaviour follows from the fact that energy is extracted from the primary oscillator and transferred to the heat bath. In what follows we will use a transmission line as a heat bath, and we prevent Poincare recurrences by ensuring that the line is appropriately initialised, and sufficiently long.

\section{INERTER-BASED HEAT BATH}

In this section we will study the embedding of damped oscillator systems of the type shown in Figure 2 in a closed system that includes a transmission-line-based heat bath. The aim is to avoid phenomenological descriptions of damping forces such as

$$
F=D_{0}\left(\dot{Q}_{0}-\dot{q}_{0}\right), \quad \text { or } \quad F=D_{0} \dot{q}_{0},
$$

where $D_{0}$ is a damping constant. This type of dissipative force precludes the use of variational modelling techniques, and thus the development of variational integration schemes. In the case of Figure 2 (a), one could use a mass-spring based transmission line [55], [56]. However, this would not work in the case of Figure 2 (b), since one of the transmission line's terminals is not 'grounded' in an inertial reference frame. For that reason we will introduce a lossless and massless transmission line comprising springs and inerters [32], which can be used to deal with either of the cases illustrated in Figure 2. 


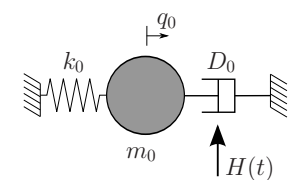

(a)

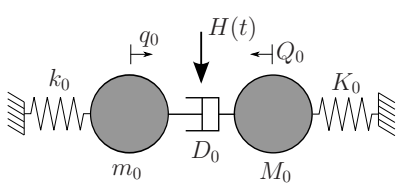

(b)

Fig. 2: (a) Damped single oscillator, and (b) damped dual oscillator systems. $H(t)$ denoting the energy back-flow to the system from the heat bath.

The inerter-based transmission line that we will study is illustrated in Figure 3. The springs are assumed linear, while

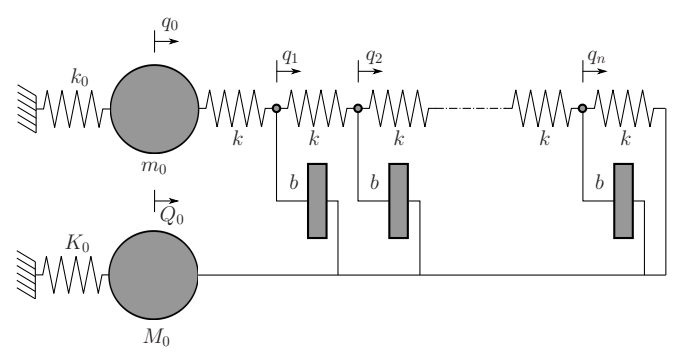

Fig. 3: Transmission line comprising springs and inerters.

the inerters produce forces that are proportional to the relative accelerations between the terminals $F_{i}=b\left(\ddot{q}_{i}-\ddot{Q}_{0}\right) ; b$ denotes the inertance. The kinetic energy stored in each inerter is given by $\frac{b}{2}\left(\dot{q}_{i}-\dot{Q}_{0}\right)^{2}$. The Lagrangian for oscillator-heat-bath system is

$$
\begin{aligned}
\mathcal{L} & =\frac{m_{0}}{2} \dot{q}_{0}^{2}+\frac{M_{0}}{2} \dot{Q}_{0}^{2}+\sum_{i=1}^{n} \frac{b}{2}\left(\dot{q}_{i}-\dot{Q}_{0}\right)^{2}-\frac{k_{0}}{2} q_{0}^{2}-\frac{K_{0}}{2} Q_{0}^{2} \\
& -\frac{k}{2}\left(q_{n}-Q_{0}\right)^{2}-\sum_{i=1}^{n} \frac{k}{2}\left(q_{i}-q_{i-1}\right)^{2},
\end{aligned}
$$

with corresponding equations of motion

$$
\begin{aligned}
m_{0} \ddot{q}_{0}+k_{0} q_{0} & =k\left(q_{1}-q_{0}\right) \\
M_{0} \ddot{Q}_{0}+K_{0} Q_{0} & =k\left(q_{n}-Q_{0}\right)+b \sum_{i=1}^{n}\left(\ddot{q}_{i}-\ddot{Q}_{0}\right) \\
b\left(\ddot{q}_{i}-\ddot{Q}_{0}\right) & =k\left(q_{i+1}-2 q_{i}+q_{i-1}\right), \\
b\left(\ddot{q}_{n}-\ddot{Q}_{0}\right) & =k\left(Q_{0}-2 q_{n}+q_{n-1}\right),
\end{aligned}
$$

where $i=1, \ldots, n-1$. Substituting (18) and (19) into (17) gives

$$
\begin{aligned}
M_{0} \ddot{Q}_{0} & +K_{0} Q_{0}=b \sum_{i=1}^{n-1}\left(\ddot{q}_{i}-\ddot{Q}_{0}\right)-k\left(q_{n}-q_{n-1}\right) \\
& =\sum_{i=1}^{n-1} k\left(q_{i+1}-2 q_{i}+q_{i-1}\right)-k\left(q_{n}-q_{n-1}\right) \\
& =k\left(q_{0}-q_{1}\right)
\end{aligned}
$$

There are a number of observations one can make about these equations:

(a) The system's initial conditions are $q_{0}(0), q_{1}(0), \cdots$, $q_{n}(0)$ and $Q_{0}(0)$, and $\dot{q}_{0}(0), \dot{q}_{1}(0), \cdots, \dot{q}_{n}(0)$ and $\dot{Q}_{0}(0)$;

(b) If the oscillators and transmission line are separated, the transmission line (18)-(19) is described by the intital conditions $q_{1}(0), \cdots, \quad q_{n}(0), \quad$ and $\dot{q}_{1}(0)$, $\cdots, \quad \dot{q}_{n}(0)$, and Neuman-type boundary conditions given by the forcing term $k\left(q_{1}-q_{0}\right)$. Once $k\left(q_{1}-q_{0}\right)$ is specified so is $k\left(q_{n}-Q_{0}\right)$, since $k\left(q_{n}-Q_{0}\right)=k\left(q_{0}-q_{1}\right)-b \sum_{i=1}^{n}\left(\ddot{q}_{i}-\ddot{Q}_{0}\right)$. The oscillators' initial conditions are $q_{0}(0), Q_{0}(0), \dot{q}_{0}(0)$, and $\dot{Q}_{0}(0)$; see (16) and (17).

(c) The forcing term $k\left(q_{1}-q_{0}\right)$ act as an interface between the oscillators and transmission line, and will be used as a basis for 'eliminating the line'.

If the number of lattice points $n$ goes to infinity, while keeping the length of the transmission line finite at $L=(n+1) \Delta x$, (15) becomes

$$
\begin{aligned}
\mathcal{L} & =\frac{m_{0}}{2}\left(\partial_{t} q(0, t)\right)^{2}-\frac{k_{0}}{2} q(0, t)^{2}+\frac{M_{0}}{2} \dot{Q}_{0}^{2}-\frac{K_{0}}{2} Q_{0}^{2} \\
& +\frac{\rho}{2} \int_{0}^{L}\left(\partial_{t} q(x, t)-\dot{Q}_{0}(t)\right)^{2} d x \\
& -\frac{K}{2} \int_{0}^{L}\left(\partial_{x} q(x, t)\right)^{2} d x
\end{aligned}
$$

in which $q(x, t)$ is the transmission line displacement, $\rho=$ $b / \Delta x$ is the inertance density of the line, and $K=k \Delta x-K$ is properly defined in the limit, while $k=K /(\Delta x)$ is not. Assuming a suitably differentiable $q(x, t)$, we can derive the Euler-Lagrange equations directly using Hamilton's principle

$$
\delta \mathcal{S}=\delta \int_{0}^{T} \mathcal{L} d t=0
$$

where $\mathcal{L}$ is the Lagrangian given in (21). Standard variational arguments and integration by parts in space and time using $\delta q(x, 0)=\delta q(x, T)=0, \delta Q_{0}(0)=\delta Q_{0}(T)=0$ (fixed boundaries in time), and $q(L, t)=Q_{0}(t)$ gives

$$
\begin{aligned}
m_{0} \partial_{t}^{2} q(0, t)+k_{0} q(0, t) & =K \partial_{x} q(0, t) \\
M_{0} \ddot{Q}_{0}(t)+K_{0} Q_{0}(t) & =\int_{0}^{L} \rho\left(\partial_{t}^{2} q(x, t)-\ddot{Q}_{0}(t)\right) d x \\
& -K \partial_{x} q(L, t) \\
\rho\left(\partial_{t}^{2} q(x, t)-\ddot{Q}_{0}(t)\right) & =K \partial_{x}^{2} q(x, t) .
\end{aligned}
$$


Substituting equations (25) into (24) gives

$$
\begin{aligned}
M_{0} \ddot{Q}_{0}(t)+K_{0} Q_{0}(t) & =\int_{0}^{L} K \partial_{x}^{2} q(x, t) d x-K \partial_{x} q(L, t) \\
& =-K \partial_{x} q(0, t)
\end{aligned}
$$

Equations (23), (25) and (26) are the spatially-distributed versions of (16), (18) and (20), respectively. The boundary forcing terms become $\pm K \partial_{x} q(0, t)$.

Remark III.1. When making detailed comparisons between Figure $2(b)$ and Figure 3, one has to ensure that total energy associated with Figure 3 is the same as that in Figure $2(b)$. For initial conditions $q_{0}(0), Q_{0}(0), \dot{q}_{0}(0)$, and $\dot{Q}_{0}(0)$, one must

1) Set $q_{0}(0)=q_{1}(0)=\cdots=q_{n}(0)=Q_{0}(0)=0$, and introduce strain energies $\frac{k_{0}}{2} q_{0}^{2}$ and $\frac{K_{0}}{2} Q_{0}^{2}$ into the oscillator springs with stiffnesses $k_{0}$ and $K_{0}$. This ensures that the oscillator strain energies are the same for both systems-there is no initial strain energy in the transmission line. In the distributed system case one would use the initial condition $q(x, 0)=0 x \in[0, L]$;

2) The oscillator mass locations are computed using the offsets $q_{0}(t):=q_{0}(t)+q_{0}(0)$, and $Q_{0}(t):=Q_{0}(t)+$ $Q_{0}(0)$

3) Set $\dot{q}_{1}(0)=\cdots=\dot{q}_{n}(0)=\dot{Q}_{0}(0)$, which ensures that the initial kinetic energies in both systems are the same, and that there is no initial kinetic energy stored in the transmission line. In the distributed system case one would use the boundary condition $\partial_{t} q(x, 0)=$ $\dot{Q}_{0}(0) x \in(0, L]$.

Conditions 1) and 2) encode initial conditions $q_{0}(0)$ and $Q_{0}(0)$ as applied to $m_{0}$ and $M_{0}$ respectively, whereas condition 3) encodes the initial conditions $\dot{q}_{0}(0)$ and $\dot{Q}_{0}(0)$ as applied to $m_{0}$ and $M_{0}$ respectively.

The following example illustrates the way in which the transmission line temporarily absorbs and stores energy, thereby producing the effects associated with linear damping. Since the oscillator-transmission system is conservative, this energy must eventually be returned and consequently the linear damping effect is only temporary; this is a manifestation of the Poincaré recurrence theorem. The time over which the line produces the effects of linear damping depends on the line's length and its wave propagation velocity. The underlying theory will be presented after the following example.

Example III.1. Figure 4 shows a numerical comparison of the influence of the phenomenological damper $D_{0}$ Figure $2(b)$ and a spatially discretized transmission line of the type shown in Figure 3. As the theory will predict, the responses are pairwise identical save for integration errors.

Figure 5 shows the propagation of the energy-transporting displacement wave. The wave is seen to 'bounce off' the far end of the transmission line at $x=L$, and then propagate backwards towards $x=0$. The effects of linear damping will cease once the reflected wave arrives at $x=0$. In this case, $\Delta x=1$, the length of the line is $L=500$ and the propagation velocity is $v=12.65$. The effects of linear damping will therefore persist for times $t \leq 2 L / v=79.0$ (see Appendix A).
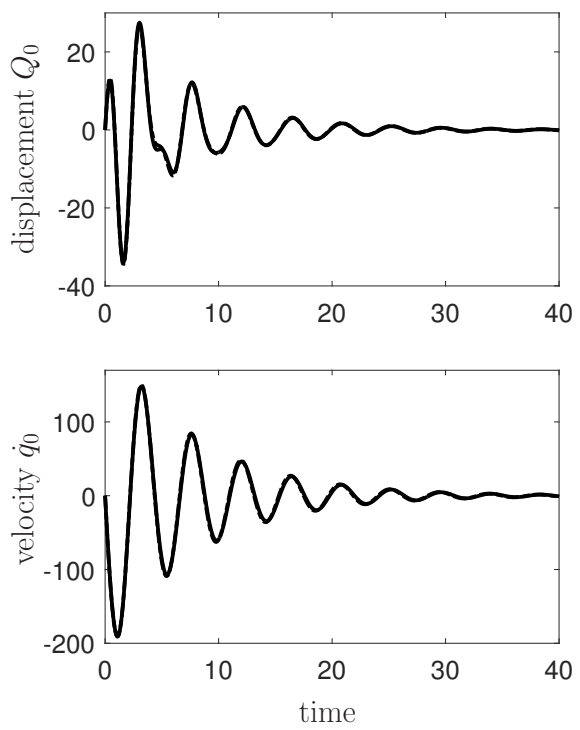

Fig. 4: Responses $Q_{0}(t)$ (top) and $\dot{q}_{0}(t)$ (bottom) of the dualoscillator system with $q_{0}(0)=150, \dot{q}_{0}(0)=0, Q_{0}(0)=0$, and $\dot{Q}_{0}(0)=50$. The closed system is shown dot-dashed, while the four-state reduced-order system is shown solid. Data: $k=1600, n=500, b=10, D_{0}=126.49, M_{0}=200$, $m_{0}=500, K_{0}=1500$ and $k_{0}=1000$.

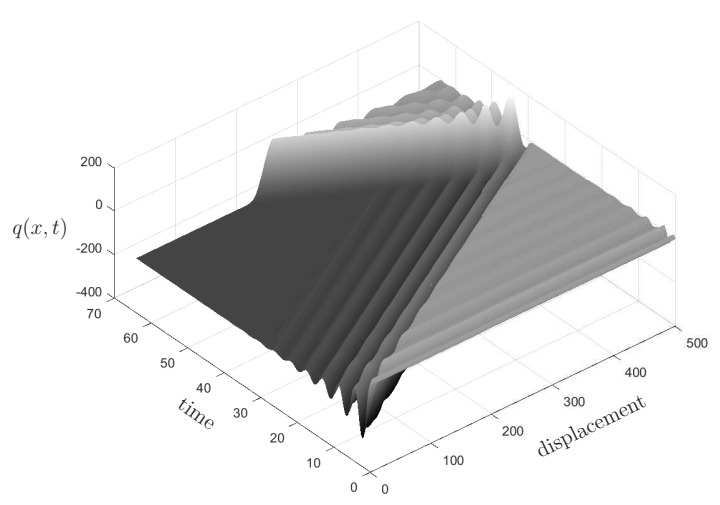

Fig. 5: Displacement wave for an inerter-based transmission line with data: $k=1600, n=500, b=10, D_{0}=126.49$, $v=12.65$.

The following theorem summarizes the properties of the system illustrated in Figure 3 in the spatially-distributed case, when the initial conditions are configured in the way described in Remark III.1.

Theorem III.1. The spatially-distributed version of the transmission line system in Figure 3 has the following properties:

(a) The displacement wave propagation velocity is $v=$ $\sqrt{K / \rho}$;

(b) The line's characteristic damping factor is given by $D_{0}=\sqrt{K \rho}$;

(c) The system in Figure $2(b)$ is described by the same set of differential equations as the system in Figure 3 prior to the arrival of the inward travelling wave at time $t_{r}=$ $2 L / v$; 
(d) If $x_{0}=\left[Q_{0}(0) \dot{Q}_{o}(0) q(0) \dot{q}(0)\right]^{T}$, the energy transferred to the line up to time $t$ is given by $x_{0}^{T} W(t) x_{0}$, where $W(t)$ is the solution of the Lyapunov equation

$$
A^{T} W(t)+W(t) A+C^{T} D_{0} C=\dot{W}(t) \quad W(0)=0 .
$$

The $A$ and $C$ matrices are given by

$$
A=\left[\begin{array}{cccc}
0 & 1 & 0 & 0 \\
\frac{-K_{0}}{M_{0}} & \frac{-D_{0}}{M_{0}} & 0 & \frac{D_{0}}{M_{0}} \\
0 & 0 & 0 & 1 \\
0 & \frac{D_{0}}{m_{0}} & \frac{-k_{0}}{m_{0}} & \frac{-D_{0}}{m_{0}}
\end{array}\right], \quad C=\left[\begin{array}{c}
0 \\
-1 \\
0 \\
1
\end{array}\right]^{T} .
$$

(e) The energy stored in the oscillator system is given by

$$
E_{\text {osc }}(t)=x_{0}^{T} \tilde{W}(t) x_{0},
$$

where $\tilde{W}(t)$ solves the Lyapunov equation

$$
A^{T} \tilde{W}(t)+\tilde{W}(t) A=\dot{\tilde{W}}(t) \quad \tilde{W}(0)=W
$$

and where $W>0^{6}$ is the solution of

$$
A^{T} W+W A+C^{T} D_{0} C=0 .
$$

(f) The closed-system stored energy is a symmetry of the system in Figure $2(b)$ and is given by $x_{0}^{T} W x_{0}$.

Proof. The wave equation (25) has a solution of the form

$$
q(x, t)=q_{+}(x-v t)+q_{-}(x+v t)+Q_{0}(t),
$$

in which $q_{+}(x-v t)$ represents an outward-bound wave, and $q_{-}(x+v t)$ and inward-bound wave. It follows by direct calculation that

$$
\begin{aligned}
& \partial_{t}^{2} q(x, t)=v^{2} q_{+}^{\prime \prime}(x-v t)+v^{2} q_{-}^{\prime \prime}(x+v t)+\ddot{Q}_{0}(t) \\
& \partial_{x}^{2} q(x, t)=q_{+}^{\prime \prime}(x-v t)+q_{-}^{\prime \prime}(x+v t)
\end{aligned}
$$

where the prime represents the derivative with respect to $\tau=$ $x \pm v t$. Substitution into the wave equation (25) gives $v=$ $\sqrt{K / \rho}$, which proves (a).

Clearly

$$
\partial_{x} q(x, t)=q_{+}^{\prime}(x-v t)+q_{-}^{\prime}(x+v t)
$$

and

$$
\partial_{t} q(x, t)=v q_{-}^{\prime}(x+v t)-v q_{+}^{\prime}(x-v t)+\dot{Q}_{0}(t) .
$$

This gives the damping force

$$
K \partial_{x} q(0, t)=\frac{K}{v}\left(2 \dot{q}_{-}(v t)+\dot{Q}_{0}(t)-\dot{q}_{0}(t)\right),
$$

where

$$
\dot{q}_{0}(t)=\partial_{t} q(0, t) .
$$

The reflected wave forcing term $H(t)=\frac{2 K}{v} \dot{q}_{-}(v t)$, as shown in Figure 2, gives rise to energy that is transferred from the transmission line back to the oscillator, which is henceforth neglected. If we substitute (33) into (23) and (26), and neglect the inward bound wave, we obtain

$$
m_{0} \ddot{q}_{0}(t)+k_{0} q_{0}(t)=K \partial_{x} q(0, t)=D_{0}\left(\dot{Q}_{0}(t)-\dot{q}_{0}(t)\right) .
$$

${ }^{6}$ Note that $(C, A)$ observable and $\operatorname{Re}\left(\lambda_{i}(A)\right)<0$.
In the same way

$M_{0} \ddot{Q}_{0}(t)+K_{0} Q_{0}(t)=-K \partial_{x} q(0, t)=-D_{0}\left(\dot{Q}_{0}(t)-\dot{q}_{0}(t)\right)$.

This completes the proof of parts (b) and (c).

Multiplying (25) by $\left(\partial_{t} q(x, t)-d_{t}\left(Q_{0}\right)\right)$, and integrating over the length of the line, gives:

$$
\begin{array}{r}
\int_{0}^{L} \rho\left(\partial_{t}^{2} q(x, t)-d_{t}^{2}\left(Q_{0}\right)\right)\left(\partial_{t} q(x, t)-d_{t}\left(Q_{0}\right)\right) d x \\
=\int_{0}^{L} K \partial_{x}^{2} q(x, t)\left(\partial_{t} q(x, t)-d_{t}\left(Q_{0}\right)\right) d x
\end{array}
$$

which is the same as

$$
\begin{array}{r}
\partial_{t} \int_{0}^{L} \frac{\rho}{2}\left(\partial_{t} q(x, t)-d_{t}\left(Q_{0}\right)\right)^{2} d x \\
=\int_{0}^{L} K \partial_{x}^{2} q(x, t)\left(\partial_{t} q(x, t)-d_{t}\left(Q_{0}\right)\right) d x .
\end{array}
$$

Integrating the right-hand side by parts gives

$$
\begin{aligned}
& \partial_{t} \int_{0}^{L} \frac{\rho}{2}\left(\partial_{t} q(x, t)-d_{t}\left(Q_{0}\right)\right)^{2} d x \\
= & \left.K \partial_{x} q(x, t)\left(\partial_{t} q(x, t)-d_{t}\left(Q_{0}\right)\right)\right|_{0} ^{L} \\
- & \frac{K}{2} \partial_{t} \int_{0}^{L}\left(\partial_{x} q(x, t)\right)^{2} d x,
\end{aligned}
$$

which becomes

$$
\begin{aligned}
& \partial_{t} \int_{0}^{L}\left(\frac{\rho}{2}\left(\partial_{t} q(x, t)-d_{t}\left(Q_{0}\right)\right)^{2}+\frac{K}{2}\left(\partial_{x} q(x, t)\right)^{2}\right) d x \\
= & -\left.K \partial_{x} q(x, t)\left(\partial_{t} q(x, t)-d_{t}\left(Q_{0}\right)\right)\right|_{x=0} \\
= & -\frac{K}{v}\left(\dot{q}_{0}-\dot{Q}_{0}\right)^{2} \\
= & D_{0}\left(\dot{q}_{0}-\dot{Q}_{0}\right)^{2},
\end{aligned}
$$

by (33) and $q(L, t)=Q_{0}(t)$. This gives

$$
\begin{aligned}
\int_{0}^{L}\left(\frac{\rho}{2}\left(\partial_{t} q(x, t)-d_{t}\left(Q_{0}\right)\right)^{2}\right. & \left.+\frac{K}{2}\left(\partial_{x} q(x, t)\right)^{2}\right) d x \\
& =\int_{0}^{t} D_{0}\left(\dot{q}_{0}-\dot{Q}_{0}\right)^{2} d \tau
\end{aligned}
$$

The left-hand side of (36) is the energy stored in the transmission line at time $t$. Since the transmission line's stored energy is initially zero, this is the same as the energy transferred from the oscillators to the transmission line up to the same time. Thus, the energy absorbed by the transmission line is equal to the energy dissipated in damper $D_{0}$ (which is the right-hand side term in (36)). The equations of motion for the system in Figure 2(b) are $\dot{x}=A x$ and

$$
\dot{q}_{0}(t)-\dot{Q}_{0}=C e^{A t} x_{0},
$$

where $A$ and $C$ are given in (28), with

$$
x_{0}=\left[\begin{array}{llll}
Q_{0}(0) & \dot{Q}_{0}(0) & q_{0}(0) & \dot{q}_{0}(0)
\end{array}\right]^{T} .
$$

This means that the power dissipated in the damper is given by

$$
P(t)=D_{0}\left(\dot{q}_{0}-\dot{Q}_{0}\right)^{2}=x_{0}^{T} e^{A^{T} t} C^{T} D_{0} C e^{A t} x_{0}
$$


with the corresponding energy dissipated (transferred to the transmission line) up to time $t$ is given by

$$
\begin{aligned}
E(t) & =\int_{0}^{t} D_{0}\left(\dot{q}_{0}-\dot{Q}_{0}\right)^{2} d \tau \\
& =x_{0}^{T} \int_{0}^{t} e^{A^{T} \tau} C^{T} D_{0} C e^{A \tau} d \tau x_{0} \\
& =x_{0}^{T} W(t) x_{0}
\end{aligned}
$$

where $W(t)$ is a solution to the Lyapunov differential equation (see page 61 [57])

$$
A^{T} W(t)+W(t) A+C^{T} D_{0} C=\dot{W}(t) \quad W(0)=0 .
$$

This completes the proof of part (d).

Since $D_{0}>0, W=\lim _{t \rightarrow \infty} W(t) \geq W(t)$ is the solution of

$$
A^{T} W+W A+C^{T} D_{0} C=0,
$$

and all the energy stored in the oscillators will be transferred to the line as $t \rightarrow \infty$. The energy in the oscillators is given by the total closed-system energy less the energy transferred to the line. Thus $E_{o s c}=x_{0}^{\prime}(W-W(t)) x_{0}$. Subtracting (38) from (39) gives (30), where $\tilde{W}(t)=W-W(t)$. This proves part (e).

The closed-system stored energy

$$
\begin{aligned}
E & =\frac{K_{0}}{2} Q^{2}(t)+\frac{M_{0}}{2} \dot{Q}^{2}(t)+\frac{k_{0}}{2} q^{2}(t)+\frac{m_{0}}{2} \dot{q}^{2}(t) \\
+ & \underbrace{\int_{0}^{L}\left(\frac{\rho}{2}\left(\partial_{t} q(x, t)-d_{t}\left(Q_{0}\right)\right)^{2}+\frac{K}{2}\left(\partial_{x} q(x, t)\right)^{2}\right) d x}_{=x_{0}^{T} W(t) x_{0}}
\end{aligned}
$$

is a conserved quantity for the system in Figure 3. The first four terms represent the energy stored in the oscillators at time $t$, while the last term represents the energy transferred to the transmission line up to time $t$. By (c) it follows that $E$ is also a symmetry for the system in Figure 2 (b).

It is easy to check that

$$
W=\operatorname{diag}\left(\frac{K_{0}}{2}, \frac{M_{0}}{2}, \frac{k_{0}}{2}, \frac{m_{0}}{2}\right) .
$$

The total energy transferred to the transmission line is thus

$E_{\infty}=x_{0}^{T} W x_{0}=\frac{K_{0}}{2} Q^{2}(0)+\frac{M_{0}}{2} \dot{Q}^{2}(0)+\frac{k_{0}}{2} q^{2}(0)+\frac{m_{0}}{2} \dot{q}^{2}(0)$, which is the initial energy. This completed the proof of part (f).

As a special case of the transmission line considered in Theorem III.1, one of the terminal masses can be held stationary, that is e.g. $Q(t)=0$, in which case the closed system shown in Figure 3 will be equivalent to the open system shown in Figure 2(a). Furthermore, in this case, all the inerters can be replaced with masses. The properties of this system are summarized in the following remark.

Remark III.2. The oscillator in Figure 2 (a) can be modelled using variational techniques and a transmission line com- prised of distributed springs and masses. The spring-mass transmission line system has the following properties:

(a) The displacement wave propagation velocity is $v=$ $\sqrt{K / \rho}$, where $\rho$ is the mass density of the line;

(b) The line's characteristic damping factor is given by $D_{0}=\sqrt{K \rho}$;

(c) The system in Figure 2 (a), with a spring-mass transmission line, is described by the same set of differential equations as the system in Figure 3 prior to the arrival of inbound travelling waves;

(d) If $x_{0}=[q(0) \dot{q}(0)]^{T}$, the energy transferred to the line up to time $t$ is given by $x_{0}^{T} W(t) x_{0}$, where $W(t)$ is the solution of (27), where the $A$ and $C$ matrices are given by

$$
A=\left[\begin{array}{cc}
0 & 1 \\
-\frac{k_{0}}{M} & -\frac{D_{0}}{M}
\end{array}\right] \quad C=\left[\begin{array}{ll}
0 & 1
\end{array}\right] .
$$

(e) The energy stored in the oscillator system is given by $E_{\text {osc }}(t)=x_{0}^{T} \tilde{W}(t) x_{0}$, where $\tilde{W}(t)$ is described by the Lyapunov equation (30), where $W$ is the solution of (31).

(f) The closed-system energy $E=x_{0}^{T} W x_{0}$, is a system symmetry.

\section{DISCRETE FORMULATION AND VARIATIONAL INTEGRATOR}

In order to illustrates the main ideas, we will make use of a simple discretization of the Lagrangian (15) on the time interval $t \in\left[t^{j}, t^{j+1}\right]$; where $j=0, \ldots, N-1$ is the discrete time index. We will use a forward difference approximation for the time derivative $\dot{q}(t) \approx \frac{q^{j+1}-q^{j}}{h}$, where $h=t^{j+1}-t^{j}$. We will also make use of a left-end-point approximation of $q(t)$; that is $q^{j} \approx q\left(t^{j}\right)$. For the closed system this discretization scheme results in

$$
\begin{aligned}
& \mathcal{L}_{d}\left(q^{j}, q^{j+1}, h\right)=h\left[\frac{m_{0}}{2}\left(\frac{q_{0}^{j+1}-q_{0}^{j}}{h}\right)^{2}\right. \\
+ & \frac{M_{0}}{2}\left(\frac{Q_{0}^{j+1}-Q_{0}^{j}}{h}\right)^{2}-\frac{k_{0}}{2}\left(q_{0}^{j}\right)^{2}-\frac{K_{0}}{2}\left(Q_{0}^{j}\right)^{2} \\
+ & \sum_{i=1}^{n} \frac{b}{2}\left(\frac{q_{i}^{j+1}-q_{i}^{j}}{h}-\frac{Q_{0}^{j+1}-Q_{0}^{j}}{h}\right)^{2} \\
- & \left.\frac{k}{2} \sum_{i=1}^{n}\left(q_{i}^{j}-q_{i-1}^{j}\right)^{2}-\frac{k}{2}\left(q_{n}^{j}-Q_{0}^{j}\right)^{2}\right] .
\end{aligned}
$$

The integration time is $T=N h$ and $\mathbf{q}^{j}=\left(q_{0}^{j}, \ldots, q_{n}^{j}, Q_{0}^{j}\right)$. It should be emphasized that this is one particular choice of a first-order variational integrator, and other schemes, including higher-order approximations, can be employed in the same way [58], [59].

The discrete Hamilton principle results in the discrete EulerLagrange equations

$$
D_{1} \mathcal{L}_{d}\left(q^{j}, q^{j+1}, h\right)+D_{2} \mathcal{L}_{d}\left(q^{j-1}, q^{j}, h\right)=0
$$


which are

$$
\begin{array}{r}
m_{0} \frac{q_{0}^{j+1}-2 q_{0}^{j}+q_{0}^{j-1}}{h^{2}}+k_{0} q_{0}^{j}=k\left(q_{1}^{j}-q_{0}^{j}\right) ; \\
M_{0} \frac{Q_{0}^{j+1}-2 Q_{0}^{j}+Q_{0}^{j-1}}{h^{2}}+K_{0} Q_{0}^{j}=k\left(q_{n}^{j}-Q_{0}^{j}\right) \\
+\sum_{i=1}^{n} \frac{b}{h^{2}}\left(q_{i}^{j+1}-2 q_{i}^{j}+q_{i}^{j-1}-\left(Q_{0}^{j+1}-2 Q_{0}^{j}+Q_{0}^{j-1}\right)\right) ; \\
\frac{b}{h^{2}}\left(q_{i}^{j+1}-2 q_{i}^{j}+q_{i}^{j-1}-\left(Q_{0}^{j+1}-2 Q_{0}^{j}+Q_{0}^{j-1}\right)\right) \\
=k\left(q_{i+1}^{j}-2 q_{i}^{j}+q_{i-1}^{j}\right) \quad i=1, \cdots, n-1, \\
\frac{b}{h^{2}}\left(q_{n}^{j+1}-2 q_{n}^{j}+q_{n}^{j-1}-\left(Q_{0}^{j+1}-2 Q_{0}^{j}+Q_{0}^{j-1}\right)\right) \\
=k\left(Q_{0}^{j}-2 q_{n}^{j}+q_{n-1}^{j}\right)
\end{array}
$$

for $j=1, \ldots, N-1$. Substituting (44) and (45) into (43) gives

$$
M_{0} \frac{Q_{0}^{j+1}-2 Q_{0}^{j}+Q_{0}^{j-1}}{h^{2}}+K_{0} Q_{0}^{j}=-k\left(q_{1}^{j}-q_{0}^{j}\right) .
$$

Equations (42), (46), (44) and (45) are respectively timediscrete approximations of (16), (20), (18) and (19).

Theorem IV.1. The discrete transmission line system in Figure 3 has the following properties:

(a) The displacement wave propagation velocity of the space-time discretized wave equation (44)-(45) is $v=$ $\sqrt{K / \rho}$;

(b) The damping force is approximated by a backward difference operator with the damping factor given by $D_{0}=\sqrt{K \rho}$

(c) Prior to the arrival of the inward travelling wave, the variationally discretized system in Figure $2(B)$ is equivalent to the variationally discretized system in Figure 3;

(d) If $x_{0}=\left[Q_{0}(0) \dot{Q}_{0}(0) q(0) \dot{q}(0)\right]^{T}$, the discrete energy transferred to the line up to time $j$ is given by $E_{d}=$ $x_{0}^{T} W_{d}(j) x_{0}$, where $W_{d}(j)$ is given by

$$
W_{d}(j)=C_{d}^{T} \frac{D_{0}}{h} C_{d}+A_{d}^{T} W_{d}(j-1) A_{d}
$$

The discrete-time state-space matrices and state vector are given by

$$
\begin{gathered}
A_{d}=\left[\begin{array}{cccc}
\frac{1}{K_{0} h} & \frac{\left(K_{0} h+D_{0}\right) h}{M_{0}} & 0 & 0 \\
0 & 0 & 0 & \frac{D_{0} h}{M_{0}} \\
0 & \frac{D_{0} h}{m_{0}} & -\frac{k_{0} h}{m_{0}} & 1-\frac{\left(k_{0} h+D_{0}\right) h}{m_{0}}
\end{array}\right], \\
0 \\
C_{d}=\left[\begin{array}{llll}
0 & -h & 0 & h
\end{array}\right] .
\end{gathered}
$$

with

$$
x_{j}^{T}=\left[\begin{array}{llll}
Q_{0}^{j} & \frac{Q_{0}^{j+1}-Q_{0}^{j}}{h} & q_{0}^{j} & \frac{q_{0}^{j+1}-q_{0}^{j}}{h}
\end{array}\right] .
$$

If $\left(C_{d}, A_{d}\right)$ is observable, and $W_{d}>0$ solves

$$
W_{d}=C_{d}^{T} \frac{D_{0}}{h} C_{d}+A_{d}^{T} W_{d} A_{d},
$$

then $\left|\lambda_{i}\left(A_{d}\right)\right|<1$, and $\lim _{j \rightarrow \infty} W_{d}(j)=W_{d}$.

(e) The discrete energy stored in the oscillator system is

$$
E_{\text {osc }}(j)=x_{0}^{T} \tilde{W}_{d}(j) x_{0},
$$

where

$$
\tilde{W}_{d}(j+1)=A_{d}^{T} \tilde{W}_{d}(j) A_{d} \quad \tilde{W}_{d}(0)=W_{d} .
$$

Proof. We again make use of the D'Alembert solution for the discretized wave equation (44), which is

$$
\begin{array}{r}
q_{i}^{j}=q_{-}\left(x_{i}+v t_{j}\right)+q_{+}\left(x_{i}-v t_{j}\right)+Q_{0}^{j} \\
\quad \text { for } \quad i=1, \ldots n, \quad j=0, \ldots, N,
\end{array}
$$

with $q_{n+1}^{j}=Q_{0}^{j}$. This solution is based on a time-space mesh that satisfies ${ }^{7} v=\frac{\Delta x}{h}$. This means that

$$
\begin{aligned}
& q_{i}^{j+1}=q_{-}\left(x_{i}+v t_{j+1}\right)+q_{+}\left(x_{i}-v t_{j+1}\right)+Q_{0}^{j+1} \\
& q_{i}^{j-1}=q_{-}\left(x_{i}+v t_{j-1}\right)+q_{+}\left(x_{i}-v t_{j-1}\right)+Q_{0}^{j-1}
\end{aligned}
$$

and

$$
\begin{aligned}
& q_{i+1}^{j}=q_{-}\left(x_{i+1}+v t_{j}\right)+q_{+}\left(x_{i+1}-v t_{j}\right)+Q_{0}^{j}, \\
& q_{i-1}^{j}=q_{-}\left(x_{i-1}+v t_{j}\right)+q_{+}\left(x_{i-1}-v t_{j}\right)+Q_{0}^{j} .
\end{aligned}
$$

Substituting $x_{i-1}=x_{i}-v h$ and $x_{i+1}=x_{i}+v h$ into (44), with $b=\rho \Delta x$ and $k=\frac{K}{\Delta x}$, we obtain

$$
v=\frac{\Delta x}{h}=\sqrt{\frac{K}{\rho}},
$$

which is the displacement wave propagation velocity for the space-time discretized wave equation (44).

Since the boundary condition $q_{n+1}^{j}=Q_{0}^{j}$ gives $q_{-}(L+$ $\left.v t_{j}\right)=-q_{+}\left(L-v t_{j}\right)$ for $j=1, \ldots, N$, and since $q_{-}\left(x_{n}+v t_{j+1}\right)=q_{-}\left(x_{n+1}+v t_{j}\right)=q_{-}\left(L+v t_{j}\right)$ and $q_{+}\left(x_{n}-v t_{j-1}\right)=q_{+}\left(x_{n+1}-v t_{j}\right)=q_{+}\left(L-v t_{j}\right)$, substituting the D'Alembert solution in equation (45) establishes (a).

Using $x_{1}=x_{0}+v h$, it follows by direct calculation that

$$
\begin{aligned}
\frac{q_{1}^{j}-q_{0}^{j}}{\Delta x} & =-\left(\frac{q_{0}^{j}-q_{0}^{j-1}}{v h}-\frac{Q_{0}^{j}-Q_{0}^{j-1}}{v h}\right. \\
& \left.-2 \frac{q_{-}\left(x_{0}+v t_{j+1}\right)-q_{-}\left(x_{0}+v t_{j-1}\right)}{2 v h}\right),
\end{aligned}
$$

where $\Delta_{t} q_{-}^{j}:=\left(q_{-}\left(x_{0}+v t_{j+1}\right)-q_{-}\left(x_{0}+v t_{j-1}\right)\right) /(2 h)$. Observing that $\Delta_{t} q_{-}$is a central difference approximation of $\frac{d}{d t} q_{-}$, it follows that (53) is a discrete version of (33).

Finally, if the reflected wave is neglected, (42) and (46) become

$$
\begin{aligned}
& m_{0} \frac{q_{0}^{j+1}-2 q_{0}^{j}+q_{0}^{j-1}}{h^{2}}+k_{0} q_{0}^{j} \\
= & -D_{0}\left(\frac{q_{0}^{j}-q_{0}^{j-1}}{h}-\frac{Q_{0}^{j}-Q_{0}^{j-1}}{h}\right), \text { and } \\
& M_{0} \frac{Q_{0}^{j+1}-2 Q_{0}^{j}+Q_{0}^{j-1}}{h^{2}}+K_{0} Q_{0}^{j} \\
= & D_{0}\left(\frac{q_{0}^{j}-q_{0}^{j-1}}{h}-\frac{Q_{0}^{j}-Q_{0}^{j-1}}{h}\right)
\end{aligned}
$$

which are discrete counterparts of (34) and (35); $D_{0}=\frac{K}{v}=$ $\sqrt{K \rho}$ is the damping constant. This establishes (b) and (c).

${ }^{7}$ Using different relations between space and time steps, i.e., $\frac{\Delta x}{h}=k v, k \in$ $\mathbb{Z}$ leads to a relabelling of the time indices in the D'Alembert solution, that is $q_{i}^{l}=q_{-}\left(x_{i}+v t_{l}\right)+q_{+}\left(x_{i}-v t_{l}\right)+Q_{0}^{l}$ with $l=k \cdot j$. 
The discrete energy $E_{d}\left(q^{j}, q^{j+1}, h\right)$ of the closed system is given by

$$
\begin{aligned}
& E_{d}\left(q^{j}, q^{j+1}, h\right)=-\frac{\partial}{\partial h} \mathcal{L}_{d}\left(q^{j}, q^{j+1}, h\right) \\
= & \frac{m_{0}}{2}\left(\frac{q_{0}^{j+1}-q_{0}^{j}}{h}\right)^{2}+\frac{M_{0}}{2}\left(\frac{Q_{0}^{j+1}-Q_{0}^{j}}{h}\right)^{2} \\
+ & \frac{k_{0}}{2}\left(q_{0}^{j}\right)^{2}+\frac{K_{0}}{2}\left(Q_{0}^{j}\right)^{2} \\
+ & \sum_{i=1}^{n} \frac{b}{2}\left(\frac{q_{i}^{j+1}-q_{i}^{j}}{h}-\frac{Q_{0}^{j+1}-Q_{0}^{j}}{h}\right)^{2} \\
- & \frac{k}{2} \sum_{i=1}^{n}\left(q_{i}^{j}-q_{i-1}^{j}\right)^{2}+\frac{k}{2}\left(q_{n}^{j}-Q_{0}^{j}\right)^{2},
\end{aligned}
$$

where the first four terms represent the energy stored in the oscillators at time $j$, while the last three terms represent the energy transferred to the transmission line up to time $j$.

Using the relation $x_{i-1}=x_{i}-v h$ and neglecting the inward travelling wave we obtain

$$
\begin{aligned}
q_{i}^{j}=q_{+}\left(x_{i}-v t_{j}\right)+Q^{j} & =q_{+}\left(x_{0}-v t_{j-i}\right)+Q^{j} \\
& =q_{0}^{j-i}+Q^{j}-Q^{j-i} .
\end{aligned}
$$

If we now substitute $q_{i}^{j}$ into (56) we obtain

$$
\begin{aligned}
& E_{d}\left(q^{j}, q^{j+1}, h\right)=\frac{m_{0}}{2}\left(\frac{q_{0}^{j+1}-q_{0}^{j}}{h}\right)^{2} \\
+ & \frac{M_{0}}{2}\left(\frac{Q_{0}^{j+1}-Q_{0}^{j}}{h}\right)^{2}+\frac{k_{0}}{2}\left(q_{0}^{j}\right)^{2}+\frac{K_{0}}{2}\left(Q_{0}^{j}\right)^{2} \\
+ & \sum_{i=1}^{\sum_{=}^{n}} \underbrace{\left(\frac{b}{2}+\frac{k h^{2}}{2}\right)}_{=D_{0} h}\left(\frac{q_{0}^{j+1-i}-q_{0}^{j-i}}{h}-\frac{Q_{0}^{j+1-i}-Q_{0}^{j-i}}{h}\right)^{2} \\
+ & \underbrace{\frac{k h^{2}}{2}}_{=}\left(\frac{q_{0}^{j-n}-Q_{0}^{j-n}}{h}\right)^{2} .
\end{aligned}
$$

Since the system displacement is zero for $t \leq 0$, we have that $q_{0}^{j-l}=0$ and $Q_{0}^{j-l}=0$ for $l \geq j$. Thus, the closed system's discrete energy is

$$
\begin{aligned}
& E_{d}\left(q^{j}, q^{j+1}\right)=\frac{m_{0}}{2}\left(\frac{q_{0}^{j+1}-q_{0}^{j}}{h}\right)^{2} \\
+ & \frac{M_{0}}{2}\left(\frac{Q_{0}^{j+1}-Q_{0}^{j}}{h}\right)^{2}+\frac{k_{0}}{2}\left(q_{0}^{j}\right)^{2}+\frac{K_{0}}{2}\left(Q_{0}^{j}\right)^{2} \\
+ & D_{0} h \sum_{i=1}^{j}\left(\frac{q_{0}^{j+1-i}-q_{0}^{j-i}}{h}-\frac{Q_{0}^{j+1-i}-Q_{0}^{j-i}}{h}\right)^{2}
\end{aligned}
$$

for $j=0, \ldots, n$. The last term represents the discrete energy dissipated in the phenomenological damper $D_{0}$ and is thus equal to the discrete energy absorbed by the transmission line up to time $j$ (last three terms in (56)). Note that the sum in (56) is an integral approximation of $\int_{0}^{t} D_{0}\left(\dot{q}_{0}(\tau)-\dot{Q}_{0}(\tau)\right)^{2} d \tau$, which is equal to the energy transferred from the oscillators to the transmission line up to time $t$ (cf. right-hand side of (36)).

In order to study further the energy transfer between the dual-oscillator system and the transmission line we will write (54) and (55) in discrete state-space form

$$
x_{j+1}=A_{d} x_{j},
$$

using the state-vector (49). The matrices given in (48) follow from (54) and (55) by direct calculation. We assume henceforth that $h$ has been chosen so that $\left|\lambda_{i}\left(A_{d}\right)\right|<1$.

The energy transferred from the oscillator system to the transmission line up to time $j$ (last term in (56)) can be written as

$$
\begin{aligned}
E_{d}^{j} & =\sum_{k=1}^{j} x_{k}^{T} C_{d}^{T} \frac{D_{0}}{h} C_{d} x_{k} \\
& =x_{0}^{T}\left(\sum_{k=1}^{j}\left(A_{d}^{T}\right)^{k} C_{d}^{T} \frac{D_{0}}{h} C_{d}\left(A_{d}\right)^{k}\right) x_{0} \\
& =x_{0}^{T} W_{d}(j) x_{0} .
\end{aligned}
$$

Since

$$
\begin{aligned}
W_{d}(j) & =\sum_{k=1}^{j}\left(A_{d}^{T}\right)^{k} C_{d}^{T} \frac{D_{0}}{h} C_{d}\left(A_{d}\right)^{k} \\
& =C_{d}^{T} \frac{D_{0}}{h} C_{d}+A_{d}^{T}\left(\sum_{k=1}^{j-1}\left(A_{d}^{T}\right)^{k} C_{d}^{T} \frac{D_{0}}{h} C_{d}\left(A_{d}\right)^{k}\right) A_{d} \\
& =C_{d}^{T} \frac{D_{0}}{h} C_{d}+A_{d}^{T} W_{d}(j-1) A_{d} \quad W(0)=0
\end{aligned}
$$

$W_{d}(j) \geq 0$ form a monotonic non-decreasing sequence, with ${ }_{2} W_{d}(j)$ the solution of the Lyapunov difference equation

$$
W_{d}(j)=C_{d}^{T} \frac{D_{0}}{h} C_{d}+A_{d}^{T} W_{d}(j-1) A_{d} \quad W(0)=0 .
$$

The asymptotic behaviour of (47) follows from well-known Lyanpunov stability theory. This proves part (d).

The discrete energy in the oscillator system is the total discrete energy, less the energy transferred to the line. Thus

$$
E_{\text {osc }}(j)=x_{0}^{\prime}\left(W_{d}-W_{d}(j)\right) x_{0} .
$$

Setting $\tilde{W}_{d}(j)=W_{d}-W_{d}(j)$, (52) follows from (47) and (50). This proves part (e).

There is no discrete analogy to part (f) of Theorem III.1, because the system's energy is not generally preserved by variational integrators. We know from backward error analysis (cf. [9]) that the total closed-system energy is 'almost conserved', i.e. the error is bounded over exponentially long time intervals using both, the closed-system variational integration scheme given by Equations (42), (46), (44) and (45), and the open-system scheme given by Equations (54) and (55); these schemes are equivalent prior to the arrival of the reflected wave. The energy decay prediction using variational integrators is thus expected to superior as compared with nonvariational schemes.

Example IV.1. For the closed system in Figure 3, suppose that $k=1600, n=5000, b=10, M_{0}=200, m_{0}=300, K_{0}=$ 
1000 and $k_{0}=1000$; the integration time step is $h=0.01$; $m_{0}$ is given initial momentum $p_{0}=20 \mathrm{Kg} \mathrm{m} / \mathrm{s}$. The initial system energy is thus $E_{0}=\frac{p_{0}^{2}}{2 m_{0}}=\frac{2}{3} \mathrm{~J}$.

Figure 6 shows the energy dissipated in $D_{0}$, which is computed using (37) and its discrete counterpart (56). In the case of the variational integrator (48) is used, while $A_{d}=I+h A$ and $A_{d}=(I-h A)^{-1}$ are used for the explicit and implicit Euler schemes. The continuous-time A-matrix is given by (28). It is clear that the variational integration scheme

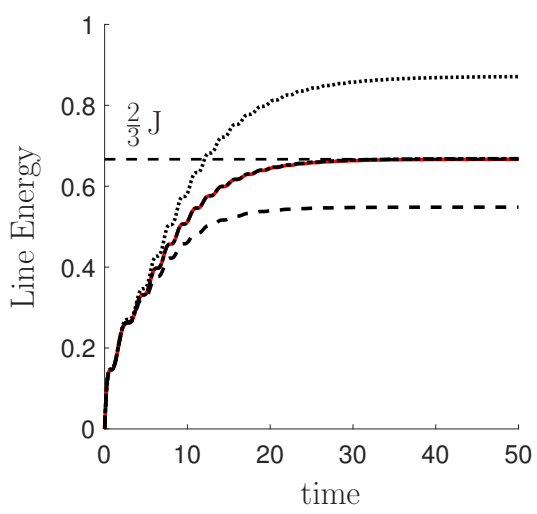

Fig. 6: Energy dissipated in $D_{0}$. The solution to (37) is shown in red. The solution to (56) for the variational scheme (48) is the dot-dash line. The implicit Euler integrator is shown dashed, while the explicit Euler integrator is shown as the dotted line.

offers superior performance as compared with the explicit and implicit Euler integrators. The inability of the explicit and implicit Euler scheme to preserve the system energy is self evident, and follows from the relatively large values of $\left\|W_{d}-W\right\|^{8}$ in the cases of the implicit and explicit Euler schemes. For this example $\left\|W_{d}-W\right\|_{2}=2.882$, 135.9, and 223.7 for the variational, implicit, and explicit Euler schemes respectively.

Figure 7 shows the energy stored in the oscillators. Again, the variational integration scheme produces a far superior record of the energy dissipation due to damping as compared with the explicit and implicit Euler schemes. The poor performance of the explicit and implicit Euler schemes is again due, primarily, to the poor initial oscillator energy estimates.

Figure 8 shows the difference in the oscillators' energy as predicted by (29), as compared with that predicted by (51) for the three integration schemes. In each case the error is dominated by the behaviour at zero time, which in turn comes from $W_{d}(h)-W$ given in (39) and (50).

The variational integrator (42), (46), (44) and (45) preserves the symplectic form associated with the closed system dynamics. Consequently, the reduced variational integrator (54) and (55), for the open system - a subsystem of the closed system - does not induce numerical dissipation in the open system dynamics in contrast to implicit and explicit Euler integration schemes. The choice of the discrete approximation

\footnotetext{
${ }^{8}$ It is well known that the solutions to (31) and (50) are equal, when $A$ and $A_{d}$, and $C$ and $C_{d}$ are related by bilinear transform [60].
}

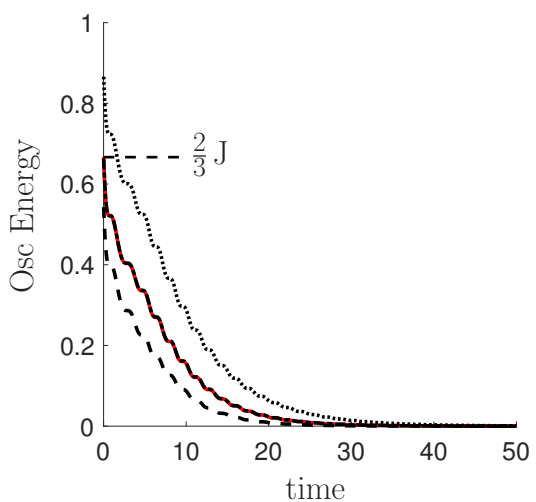

Fig. 7: Energy stored in the oscillators. The theoretical response computed using (29) is shown in red. The variational integrator (51) shown as a dot-dash line. The implicit Euler integrator is shown dashed, while the explicit Euler integrator is shown dotted.

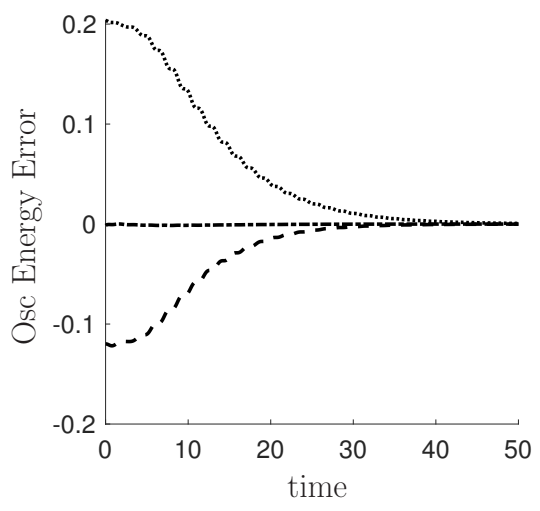

Fig. 8: Error in the predicted stored oscillator energy. The variational integrator shown as a dot-dash line. The implicit Euler integrator is shown dashed, while the explicit Euler integrator is shown dotted.

$\mathcal{L}_{d}$ of the action determines the convergence order of the integrator (see [7], [58], [59]). In this case, the variational integrator is first order, which is inherited from the firstorder approximation of the Lagrangian (41); Higher-order variational integrators can be constructed using higher-order approximations of the Lagrangian. The given choice of $\mathcal{L}_{d}$ produces a fast semi-explicit integration scheme given by (54) and (55)). As is demonstrated in future work, the benefits of variational schemes are far greater for nonlinear dissipation mechanisms.

\section{Central Force PRoblems}

Suppose that particles $m_{1}$ and $m_{2}$ are subject only to forces of mutual attraction (see [36] pages 70-72). These central forces might be gravitational, electrostatic, spring or damping forces (see Figure 9). In the celestial mechanics context it follows from Newton's laws that:

$$
\begin{aligned}
& m_{1} \ddot{\mathbf{x}}_{1}=\mathbf{f}\left(\mathbf{x}_{1}-\mathbf{x}_{2}\right) \\
& m_{2} \ddot{\mathbf{x}}_{2}=-\mathbf{f}\left(\mathbf{x}_{1}-\mathbf{x}_{2}\right) .
\end{aligned}
$$




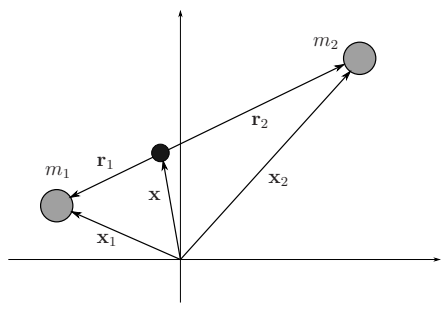

Fig. 9: The particles $m_{i}$ are located at $\mathbf{x}_{i}$ with respect to an inertial frame, $\mathbf{x}$ locates the mass centre of the two-body system, and $\mathbf{r}_{i}$ locate the particles relative to the centre of mass.

Define

$$
\mathbf{r}=\mathbf{r}_{1}-\mathbf{r}_{2}=\mathbf{x}_{1}-\mathbf{x}_{2},
$$

with the system's mass centre is located at

$$
m_{1} \mathbf{x}_{1}+m_{2} \mathbf{x}_{2}=\left(m_{1}+m_{2}\right) \mathbf{x} .
$$

Equations (57) and (58) decouple to yield

$$
\begin{aligned}
\left(m_{1}+m_{2}\right) \ddot{\mathbf{x}} & =0 \\
\ddot{\mathbf{r}} & =\left(\frac{1}{m_{1}}+\frac{1}{m_{2}}\right) \mathbf{f}(\mathbf{r}) .
\end{aligned}
$$

Equation (61) shows that in the absence of external forces the motion of the centre of mass is unaccelerated. Equation (62) establishes that $\mathbf{r}$ moves as if it refers to the motion of a particle of mass $\mu$ :

$$
\mu=\frac{m_{1} m_{2}}{m_{1}+m_{2}} .
$$

Such problems include the Kepler problem and its variants [61]-[63]. In the case of the Kepler problem with linear damping, a high-order conservative model can be contemplated using an inerter-based transmission line heat bath. This type of transmission line will introduce linear damping, and because it is massless, it will not introduce extraneous force terms.

Consider the Kepler problem with a force term $\alpha \dot{\mathbf{r}}$ that is proportional to the velocity:

$$
\ddot{\mathbf{r}}+\alpha \dot{\mathbf{r}}+\frac{\mu \mathbf{r}}{r^{3}}=0
$$

in which $\alpha$ and $\mu$ are constants [61]-[63].

Cross multiplying (63) on the left by $\mathbf{r}$ gives

$$
\begin{aligned}
0 & =\mathbf{r} \times \ddot{\mathbf{r}}+\alpha \mathbf{r} \times \dot{\mathbf{r}} \\
& =\dot{\mathbf{H}}+\alpha \mathbf{H},
\end{aligned}
$$

where $\mathbf{H}=\mathbf{r} \times \dot{\mathbf{r}}$ is the angular momentum vector. The vector product of (64) with $\mathbf{H}$ gives

$$
\mathbf{H} \times \dot{\mathbf{H}}=0,
$$

which shows that $\dot{\mathbf{H}}$ and $\mathbf{H}$ are collinear. This means that the direction of the angular momentum vector is conserved, although its magnitude is not. Since $\hat{\mathbf{H}}=\frac{\mathbf{H}}{H}$, where $H$ is the magnitude of the angular momentum, is perpendicular to both $\mathbf{r}$ and $\dot{\mathbf{r}}$, the constancy of $\hat{\mathbf{H}}$ implies that a planar orbit is obtained as in the classical problem formulation.
From (64) one sees that

$$
(\dot{H}+\alpha H) \hat{\mathbf{H}}=0
$$

and so

$$
\dot{H}+\alpha H=0,
$$

which has solution

$$
H=H_{0} e^{-\alpha t},
$$

in which $H_{0}=H(0)$ is magnitude of the angular momentum at $t=0$.

Hamilton and Laplace-Runge-Lenz like vectors can now be defined that are analogues of the Hamilton and LaplaceRunge-Lenz vectors for the classical Kepler problem [63]. It follows from (65) and (63) that

$$
\ddot{\mathbf{r}}-\frac{\dot{H}}{H} \dot{\mathbf{r}}+\frac{\mu \mathbf{r}}{r^{3}}=0
$$

which gives

$$
\frac{d}{d t}\left(\frac{\dot{\mathbf{r}}}{H}\right)+\frac{\mu \hat{\mathbf{r}}}{H r^{2}}=0 .
$$

Thus

$$
\mathbf{K}=\frac{\dot{\mathbf{r}}}{H}+\int \frac{\mu \hat{\mathbf{r}}}{H r^{2}} d t
$$

is constant, which is a vector first integral that serves as a generalisation of Hamilton's vector for the classical Kepler problem. An analogue of the Laplace-Runge-Lenz vector can be found by multiplying $\mathbf{K}$ by the constant $\hat{\mathbf{H}}$ to obtain

$$
\mathbf{J}=\mathbf{K} \times \hat{\mathbf{H}}=\frac{\dot{\mathbf{r}} \times \hat{\mathbf{H}}}{H}+\int \frac{\mu \hat{\mathbf{r}} \times \hat{\mathbf{H}}}{H r^{2}} d t,
$$

which is constant and is illustrated in Figure 10. The Laplace-

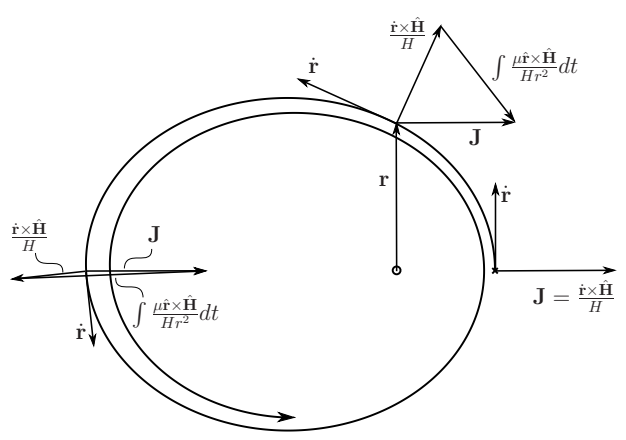

Fig. 10: Analog of the Laplace-Runge-Lenz vector for the linearly damped Kepler problem; adapted from Figure 3.18 in [36] for the classical case.

Runge-Lenz analog lies in the orbital plane and points generally in the direction of the major axis of the elliptical orbit.

\section{A. Lagrangian for Kepler problem with transmission line}

Since the direction of angular momentum is preserved, we consider planar motion only. Suppose that $\mathbf{r}=\left(x_{1}, x_{2}\right) \in$ $\mathbb{R}^{2}$ and $r=|\mathbf{r}|=\sqrt{\left(x_{1}^{2}+x_{2}^{2}\right)}$ in a rectangular coordinate system. Assume that there is an inerter-based transmission line connecting the orbiting planet to the origin with displacement coordinates $\mathbf{r}(x, t)$, where $\mathbf{r}(0, t)$ is the position of the orbiting 
planet and that $\mathbf{r}(L, t)$ is connected to the origin. Then the Lagrangian is

$$
\begin{aligned}
\mathcal{L} & =\frac{M}{2}\left(\partial_{t} \mathbf{r}(0, t)\right)^{2}+\frac{\rho}{2} \int_{0}^{L}\left(\partial_{t} \mathbf{r}(x, t)\right)^{2} d x \\
& +\frac{\mu M}{r(0, t)}-\frac{K}{2} \int_{0}^{L}\left(\partial_{x} \mathbf{r}(x, t)\right)^{2} d x,
\end{aligned}
$$

with $\rho=b / \Delta x$ and $K=k \Delta x$. If $\mathbf{r}(x, 0), \mathbf{r}(x, T)$ and $\mathbf{r}(L, t)$ are fixed, then the Euler-Lagrange equations are

$$
\begin{aligned}
\partial_{t}^{2} \mathbf{r}(0, t)+\frac{\mu \mathbf{r}(0, t)}{r^{3}} & =\frac{K}{M} \partial_{x} \mathbf{r}(0, t) \\
\rho \partial_{t}^{2} \mathbf{r}(x, t) & =K \partial_{x}^{2} \mathbf{r}(x, t)
\end{aligned}
$$

where (67) is a wave equation in the $x_{1}$ and $x_{2}$ dimensions. The solution of the initially quiescent equation is

$$
\mathbf{r}(x, t)=\mathbf{r}_{+}\left(t-\frac{x}{v}\right)+\mathbf{r}_{-}\left(t+\frac{x}{v}\right) .
$$

The space-discretized version of (66) is

$$
\mathcal{L}=\frac{M}{2} \dot{\mathbf{r}}_{0}^{2}+\sum_{i=1}^{n} \frac{b}{2} \dot{\mathbf{r}}_{i}^{2}+\frac{\mu M}{r_{0}}-\frac{k}{2} r_{n}^{2}-\sum_{i=1}^{n} \frac{k}{2}\left|\mathbf{r}_{i}-\mathbf{r}_{i-1}\right|^{2}
$$

where $\mathbf{r}_{i}=\left(\left(x_{1}\right)_{i},\left(x_{2}\right)_{i}\right)$ and $r_{i}=\left|\mathbf{r}_{i}\right|=\sqrt{\left(x_{1}\right)_{i}^{2}+\left(x_{2}\right)_{i}^{2}}$ with the corresponding equations of motion

$$
\begin{aligned}
M \ddot{\mathbf{r}}_{0} & =-\frac{\mu M \mathbf{r}_{0}}{r_{0}^{3}}+k\left(\mathbf{r}_{1}-\mathbf{r}_{0}\right) \\
b \ddot{\mathbf{r}}_{i} & =k\left(\mathbf{r}_{i+1}-2 \mathbf{r}_{i}+\mathbf{r}_{i-1}\right), i=1, \ldots, n-1 \\
b \ddot{\mathbf{r}}_{n} & =k\left(-2 \mathbf{r}_{n}+\mathbf{r}_{n-1}\right) .
\end{aligned}
$$

Using the line elimination process, and neglecting the reflected wave, the equation of motion is

$$
M \ddot{\mathbf{r}}(0, t)+D_{0} \dot{\mathbf{r}}(0, t)+\frac{M \mu \mathbf{r}(0, t)}{r^{3}}=0 .
$$

Figure 11 illustrates the damped Kepler orbits computed with explicit (dotted), implicit (dashed) and variational (dotdashed) Euler schemes with integration time step $h=0.001$. The variational integration scheme is indistinguishable from a high-order Runge-Kutta scheme (red), whereas the explicit and implicit Euler schemes show significant drift with the implicit scheme the least satisfactory of the three.

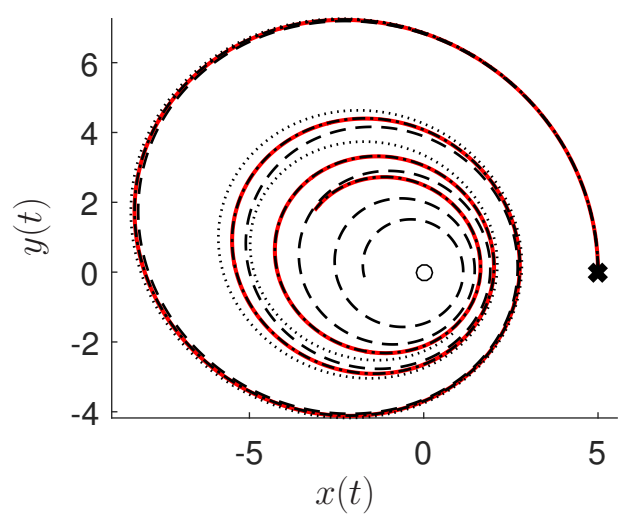

Fig. 11: Damped Kepler orbits for initial conditions $\mathbf{q}(0)=$ $\left[\begin{array}{ll}5 & 0\end{array}\right]^{T}$ and $\mathbf{p}(0)=\left[\begin{array}{ll}0 & 17\end{array}\right]^{T}$.
Figure 12 shows the planetary energy and angular momentum magnitude for the damped Kepler problem with the initial conditions, integration time step, and nomenclature adopted in Figure 11. The analytic energy and angular momentum (red) are indistinguishable from the variational Euler scheme (dotdash).
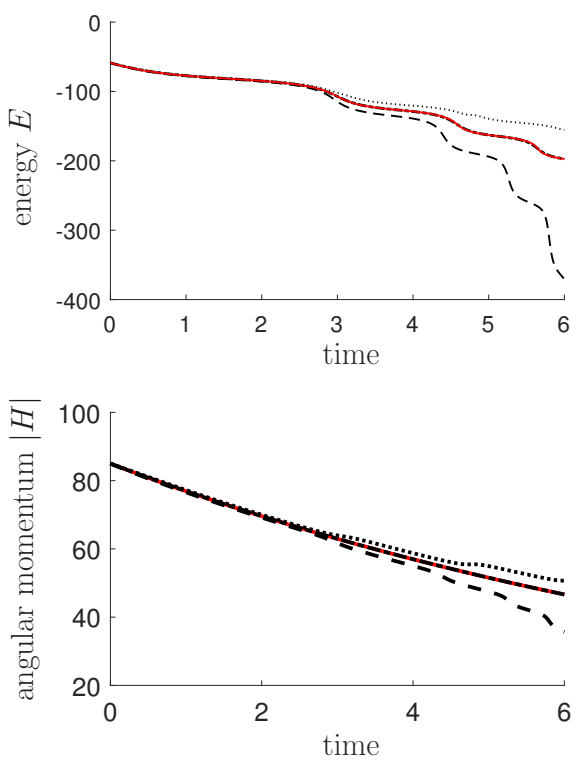

Fig. 12: (a) planetary energy, and (b) planetary angular momentum for the damped Kepler problem.

We conclude this section with a study of the magnitude behaviour of the analogue of the Laplace-Runge-Lenz vector for the damped Kepler orbits described in Figure 11. Figure 13 shows the analytic solution (red), which is constant according to the presented theory; the classical (undamped) case LaplaceRunge-Lenz vector is constant too. The variational Euler scheme (dot-dash) is indistinguishable from it. The explicit (dotted) and implicit (dashed) Euler schemes show significant variability, with the implicit scheme the least accurate of the two.

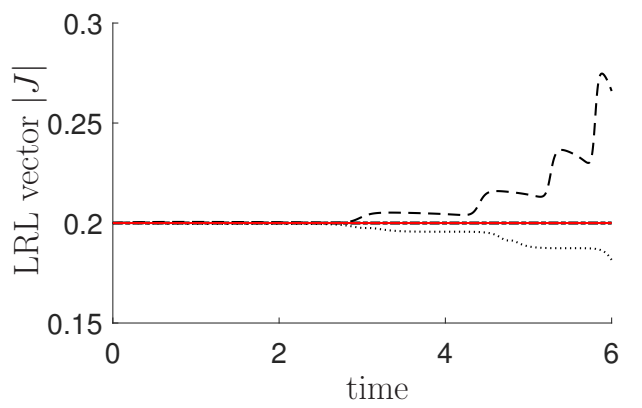

Fig. 13: Magnitude of the analogue of the Laplace-Runge-Lenz vector for the damped Kepler orbits described in Figure 11.

\section{CONCLUSIONS}

The equations of motion for systems involving dissipation cannot be derived using Hamilton's principles-this represents a long-standing difficulty with in classical mechanics. Our 
introductory comments refer to several approaches to the possible solution of this problem-many of which rely on some form of state dimension doubling. We make use of a springinerter based transmission line system as a heat absorbing environment, which is akin to classical notion of immersing an open dissipative system in a heat bath in order to form a high-order (closed) conservative system that is describable by variational methods. This approach appears initially to involve a substantial inflation of the state-space. However, as with the Caldeira-Leggett system, the interface conditions between the system and reservoir allow the heat bath to be eliminated.

The results of this paper appear to offer a useful and general way of developing variational integration schemes for systems with dissipation. As with many of the papers in the physics literature on this topic, we use a dual-oscillator system as a demonstration example. In essence, the transmission line acts as an 'energy sponge' that mimics damping influences. As with all sponges, the transmission system eventually returns the stored energy from whence it came. Since our closed system is conservative, the Poincare recurrence theorem ensures that the state returns to a state very close to the initial state; this is achieved physically by reflections in the transmission system.

Several examples, including a substantial nonlinear one, are used to illustrate the utility of this approach in deriving variational integrators, which may form a useful component of a general-purpose numerical optimal control solver for problems involving electro-mechanical systems.

\section{APPENDIX A}

We have already established that the wave equation (25) has a solution of the form (32), where $q_{+}(x-v t), q_{-}(x+$ $v t)$, and $Q_{0}(t) \equiv q(L, t)$, denote the right-travelling wave, the left-travelling wave, and the position of the second oscillator respectively.

Suppose that the initial conditions are

$$
\begin{aligned}
& q(x, t=0)=f(x) \quad x \in[0, L] \\
& \partial_{t} q(x, t=0)=g(x) \quad x \in[0, L],
\end{aligned}
$$

while the Neumann-type boundary conditions are

$$
\begin{aligned}
\partial_{x} q(x=0, t) & =h_{1}(t) \\
\partial_{x} q(x=L, t) & =h_{2}(t) .
\end{aligned}
$$

It is easy to check using the equation of motion (24) and (25) that

$$
h_{2}(t)=h_{1}(t)+\int_{0}^{L} \varrho\left(\partial_{t}^{2} q(x, t)-\ddot{Q}_{0}(t)\right) d x .
$$

Knowledge of $h_{1}(t)$ and the initial conditions $f(x)$ and $g(x)$ determines the second term in (70), which specifies $h_{2}(t)$. The three functions $\left\{f(x), g(x), h_{1}(t)\right\}$ thus encode the initial and boundary conditions of the system.

We now study (32) with the initial and boundary conditions given in equations (68) and (69). The wave equation solution can be expressed in terms of temporal and spatial derivatives as follows

$$
\begin{aligned}
q_{-}^{\prime}(x+v t) & =\frac{1}{2} \partial_{x} q(x, t)+\frac{1}{2 v}\left(\partial_{t} q(x, t)-\dot{Q}_{0}(t)\right) \\
q_{+}^{\prime}(x-v t) & =\frac{1}{2} \partial_{x} q(x, t)-\frac{1}{2 v}\left(\partial_{t} q(x, t)-\dot{Q}_{0}(t)\right)
\end{aligned}
$$

We can express (71) as

$$
\begin{aligned}
& q_{-}^{\prime}(x)=\frac{1}{2 v}\left(a \delta(x)+b \theta(x)-\dot{Q}_{0}(0)\right)=0 \quad x \in(0, L) \\
& q_{+}^{\prime}(x)=\frac{-1}{2 v}\left(a \delta(x)+b \theta(x)-\dot{Q}_{0}(0)\right)=0 \quad x \in(0, L)
\end{aligned}
$$

using $f(x)=0$, and $g(x)=a \delta(x)+b \theta(x)$. This shows that for these initial conditions there are no travelling waves at $t=0$ in the domain $x \in(0, L)$.

Summing equations (71) at $x=0$ gives

$$
q_{-}^{\prime}(v t)+q_{+}^{\prime}(-v t)=\partial_{x} q(0, t)
$$

or what is the same

$$
q_{-}^{\prime}(x)+q_{+}^{\prime}(-x)=\partial_{x} q(0, t) .
$$

Since $q_{-}^{\prime}(x)=0$ for $x \in(0, L)$

$$
q_{+}^{\prime}(-x)=\partial_{x} q(0, t) \quad x \in(0, L) .
$$

Condition (73) shows that there is a right-travelling wave in the extended domain $(-L, 0)$ at $t=0$ as depicted below:

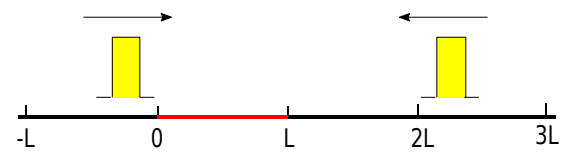

At $x=L$ :

$$
q(L, t) \equiv Q_{0}(t)=q_{+}(L-v t)+q_{-}(L+v t)+Q_{0}(t)
$$

thus

and

$$
q_{+}(L-x)+q_{-}(L+x)=0
$$

$$
-q_{+}^{\prime}(L-x)+q_{-}^{\prime}(L+x)=0 .
$$

According to (72) $q_{+}^{\prime}(x)=0$ for $x \in(0, L)$, while (74) implies that there is no left-travelling wave in the domains $(L, 2 L)$. Finally, (74) shows that there is a left-travelling wave in the domain $(2 L, 3 L)$ at $t=0$, that will reach $x=0$ at $t_{r}=2 L / v$.

\section{ACKNOWLEDGEMENT}

We are indebted to Fernando Jimenez Alburquerque for several helpful suggestions.

\section{REFERENCES}

[1] J. T. Betts, Practical methods for Optimal Control and estimation Using Nonlinear Programming, 2nd ed. Philadelphia, PA: SIAM, 2001.

[2] S. Ober-Blöbaum, O. Junge, and J. E. Marsden, "Discrete mechanics and optimal control: an analysis," ESAIM: COCV, vol. 17, pp. 322-352, 2011.

[3] C. Kane, J. E. Marsden, and M. Ortiz, "Symplectic-energy-momentum preserving variational integrators," J. Math Physics, vol. 40, no. 7, pp. 3353-3371, 1999. 
[4] J. M. Sanz-Serna, "Symplectic integrators for Hamiltonian problems: an overview," Acta Numerica, vol. 1, pp. 243-286, 1991.

[5] A. Moore, S. Ober-Blöbaum, and J. E. Marsden, "Trajectory design combining invariant manifolds with discrete mechanics and optimal control," Journal of Guidance Control and Dynamics, vol. 35, no. 5, pp. 1507-1525, 2012.

[6] R. Serban, W. S. Koon, M. W. Lo, J. E. Marsden, L. R. Petzold, S. D. Ross, and R. S. Wilson, "Halo orbit mission correction maneuvers using optimal control," Automatica, vol. 38, pp. 571-583, 2002.

[7] J. Marsden and M. West, "Discrete mechanics and variational integrators," Acta Numer., vol. 10, pp. 357-514, 2001.

[8] A. Lew, J. E. Marsden, M. Ortiz, and M. West, "An overview of variational integrators," in Finite Element Methods: 1970's and Beyond, L. P. Franca, T. E. Tezduyar, and A. Masud, Eds. CIMNE, 2004, pp. 98-115.

[9] E. Hairer, C. Lubich, and G. Wanner, Geometric Numerical Integration: Structure-Preserving Algorithms for Ordinary Differential Equations, 2nd ed., ser. Comput. Math., vol. 31. Springer-Verlag, 2006.

[10] A. M. Bloch, Nonholonomic mechanics and control. Springer, 2003.

[11] C. Kane, J. E. Marsden, M. Ortiz, and M. West, "Variational integrators and the Newmark algorithm for conservative and dissipative mechanical systems," International Journal for Numerical Methods in Engineering, vol. 49, no. 10, pp. 1295-1325, 2000.

[12] A. Lew, J. E. Marsden, M. Ortiz, and M. West, "Variational time integrators," International Journal for Numerical Methods in Engineering, vol. 60 , no. 1 , pp. 153-212, 2004

[13] S. Ober-Blöbaum, O. Junge, and J. E. Marsden, "Discrete mechanics and optimal control: an analysis," Control, Optimisation and Calculus of Variations, vol. 17, no. 2, pp. 322-352, 2011.

[14] K. Modin and G. Söderlind, "Geometric integration of hamiltonian systems perturbed by Rayleigh damping," BIT Numerical Mathematics, vol. 51, no. 4, pp. 977-1007, Dec 2011.

[15] S. Leyendecker, J. E. Marsden, and M. Ortiz, "Variational integrators for constrained dynamical systems," Journal of Applied Mathematics and Mechanics, vol. 88, no. 9, pp. 677-708, 2008.

[16] S. Leyendecker, S. Ober-Blöbaum, J. E. Marsden, and M. Ortiz, "Discrete mechanics and optimal control for constrained systems," Optimal Control, Applications and Methods, vol. 31, no. 6, pp. 505-528, 2010.

[17] M. Kobilarov, J. E. Marsden, and G. S. Sukhatme, "Geometric discretization of nonholonomic systems with symmetries," Discrete and Continuous Dynamical Systems - Series S, vol. 1, no. 1, pp. 61-84, 2010.

[18] J. Cortés and S. Martínez, "Non-holonomic integrators," Nonlinearity, vol. 14, no. 5, pp. 1365-1392, 2001.

[19] R. C. Fetecau, J. E. Marsden, M. Ortiz, and M. West, "Nonsmooth Lagrangian Mechanics and Variational Collision Integrators," SIAM Journal on Applied Dynamical Systems, vol. 2, no. 3, pp. 381-416, 2003.

[20] N. Bou-Rabee and H. Owhadi, "Stochastic variational integrators," IMA Journal on Numerical Analysis, vol. 29, pp. 421-443, 2008.

[21] M. Tao, H. Owhadi, and J. E. Marsden, "Nonintrusive and structure preserving multiscale integration of stiff ODEs, SDEs, and Hamiltonian systems with hidden slow dynamics via flow averaging," Multiscale Modeling and Simulation, vol. 8, no. 4, pp. 1269-1324, 2010.

[22] S. Leyendecker and S. Ober-Blöbaum, "A variational approach to multirate integration for constrained systems," in Multibody Dynamics, ser. Computational Methods in Applied Sciences, J.-C. Samin and P. Fisette, Eds. Springer Netherlands, 2013, vol. 28, pp. 97-121.

[23] A. Stern and E. Grinspun, "Implicit-explicit variational integration of highly oscillatory problems," SIAM Multiscale Modeling and Simulation, vol. 7, pp. 1779-1794, 2009.

[24] A. Lew, J. E. Marsden, M. Ortiz, and M. West, "Asynchronous variational integrators," Archive for Rational Mechanics and Analysis, vol. 167, pp. 85-146, 2003.

[25] J. E. Marsden, G. W. Patrick, and S. Shkoller, "Multisymplectic geometry, variational integrators, and nonlinear PDEs," Communication in Mathematical Physics, vol. 199, pp. 351-395, 1998.

[26] D. Furihata and T. Matsuo, Discrete Variational Derivative Method: A Structure-Preserving Numerical Method for Partial Differential Equations. Chapman and Hall/CRC, 2010.

[27] S. Ober-Blöbaum, M. Tao, M. Cheng, H. Owhadi, and J. E. Marsden, "Variational integrators for electric circuits," Journal of Computational Physics, vol. 242, pp. 498-530, 2013.

[28] C. M. Campos, S. Ober-Blöbaum, and E. Trélat, "High order variational integrators in the optimal control of mechanical systems," Discrete and Continuous Dynamical Systems, vol. 35, no. 9, pp. 4193-4223, 2015.
[29] H. Dekker, "Classical and quantum mechanics of the damped harmonic oscillator," Physics Reports, vol. 80, no. 1, pp. 1-110, 1981.

[30] R. Kubo, "The fluctuation-dissipation theorem," Reports on Progress in Physics, vol. 29, no. Part 1, 1966.

[31] J. L. Anderson, Foundations of general relativity. Academic Press, 1967 , ch. 10 , p. 344.

[32] M. C. Smith, "Synthesis of mechanical networks: the inerter," IEEE Transactions on Automatic Control, vol. 47, no. 10, pp. 1648-1662, 2002.

[33] D. G. Kirchhoff, "Über die Anwendbarkeit der Formeln für die Intensitäten der galvanischen Ströme in einem System linearer Leiter auf Systeme, die zum Theil aus nicht linearen Leitern bestehen." Ann. Phys., vol. 75, pp. 189-205, 1848.

[34] J. C. Maxwell, A treatise on Electricty and Magnetism. McMillan and Co, 1873 , vol. 1 .

[35] C. Cherry, "Some general theorems for non-linear systems possessing reactance," The London, Edinburgh, and Dublin Philosophical Magazine and Journal of Science, vol. 42, no. 333, pp. 1161-1177, 1951, series 7.

[36] H. Goldstein, C. Poole, and J. Safko, Classical Mechanics, 3rd ed. Addison Wesley, 2001.

[37] E. Kanai, "On the quantization of the dissipative system," Progress in Theoretical Physics, vol. 3, no. 4, pp. 440-442, 1948.

[38] P. Caldirola, "Forze non conservative nella meccanica quantistica," Il Nuovo Cimento, vol. 18, no. 9, pp. 393-400, 1941.

[39] J. R. Ray, "Lagrangians and systems they describe - how not to treat dissipation in quantum mechanics," American Journal of Physics, vol. 47, no. 7, pp. 626-629, 1979.

[40] H. Bateman, "On dissipative systems and related variational principles," Physical Review, vol. 38, pp. 815-819, 1931.

[41] F. H. and T. Y., "Quantization of the damped oscillator ( festschrift for i. i. rabi)," Trans. New York Acad. Sci., vol. 38, pp. 44-53, 1977.

[42] P. M. Morse and H. Feshbach, Methods of Theoretical Physics. New York: McGraw-Hil, 1953.

[43] D. M. Greenberger, "A critique of the major approaches to damping in quantum theory," Journal of Mathematical Physics, vol. 20, no. 5, pp. 762-770, 1979.

[44] C. R. Galley, "Classical mechanics of nonconservative systems," Phys. Rev. Lett., vol. 110, pp. 174301-(1-5), 2013.

[45] D. M. de Diego and R. S. M. de Almagro, "Variational order for forced lagrangian systems," Nonlinearity, vol. 31, pp. 3814-3846, 2018.

[46] P. S. Bauer, "Dissipative dynamical systems," Proc Natl Acad Sci, vol. 17, pp. 311-314, 1931.

[47] F. Riewe, "Nonconservative Lagrangian and Hamiltonian mechanics," Phys. rev. E, vol. 53, no. 2, pp. 1890-1899, 1996.

[48] - "Mechanics with fractional derivatives," Physical Review E, vol. 55, no. 3, pp. 3581-3592, 1997.

[49] J. Cresson and P. Inizan, "Variational formulations of differential equations and asymmetric fractional embedding," Journal of Mathematical Analysis and Applications, vol. 385, no. 2, pp. 975 - 997, 2012.

[50] F. Jiménez and S. Ober-Blöbaum, "A fractional variational approach for modelling dissipative mechanical systems:continuous and discrete settings," IFAC-PapersOnLine, vol. 51, no. 3, pp. 50 - 55, 2018, 6th IFAC Workshop on Lagrangian and Hamiltonian Methods for Nonlinear Control LHMNC 2018.

[51] A. O. Caldeira and A. J. Leggett, "Path integral approach to Brownian motion," Physica A, vol. 121, pp. 587-616, 1983.

[52] R. Zwanzig, "Nonlinear generalized Langevin equations," Journal of Statistical Physics, vol. 9, no. 3, pp. 215-220, 1973.

[53] G. W. Ford and M. Kac, "On the quantum Langevin equation," Journal of Statistical Physics, vol. 46, no. 5-6, pp. 803-810, 1987.

[54] G.-L. Ingold, Coherent Evolution in Noisy Environments, ser. Lecture Notes in Physics. Springer, 2002, ch. 1, p. 31.

[55] B. Yurke, "Conservative model for the damped harmonic oscillator," American Journal of Physics, vol. 52, pp. 1099-1102, 1984

[56] - "Quantizing the damped harmonic oscillator," American Journal of Physics, vol. 54, pp. 1133-1139, 1986.

[57] R. W. Brockett, Finite Dimensional Linear Systems. New York, London: John Wiley and Sons, Inc., 1970.

[58] S. Ober-Blöbaum and N. Saake, "Construction and analysis of higher order Galerkin variational integrators," Advances in Computational Mathematics, vol. 41, no. 6, pp. 955-986, 2015.

[59] S. Ober-Blöbaum, "Galerkin variational integrators and modified symplectic Runge-Kutta methods," IMA Journal of Numerical Analysis, vol. 37, no. 1, pp. 375-406, 2017.

[60] M. Green and D. J. N. Limebeer, Linear Robust Control. Prentice Hall, 1995. 
[61] P. G. L. Leach, "The first integrals and orbit equation for the Kepler problem with drag," J. Phys. A: Math. Gen., vol. 20, pp. 1997-2002, 1987.

[62] V. M. Gorringe and P. G. L. Leach, "Hamilton-like vectors for a class of kepler problems with a force proportional to velocity," Celestial Mechanics, vol. 41, pp. 125-130, 1988.

[63] P. G. L. Leach and G. P. Flessas, "Generalisations of the Laplace-RungeLenz vector," Journal of Nonlinear Mathematical Physics, vol. 10, no. 3, pp. 340-423, 2003.

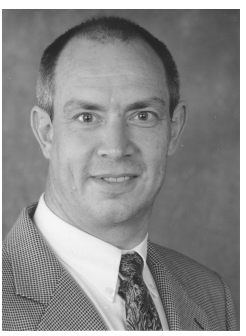

David. J. N. Limebeer received a B.Sc.(Eng) degree from the University of the Witwatersrand in 1974, $\mathrm{MSc}(\mathrm{Eng})$ and $\mathrm{PhD}$ degrees from the University of Natal in 1977 and 1980, respectively, and the DSc (Eng) from the University of London in $1992 . \mathrm{He}$ was a post-doc researcher at the University of Cambridge between 1980 and 1984. He then joined the Electrical and Electronic Engineering Department at Imperial College as a lecturer. He was promoted to Reader in 1989, Professor in 1993, Head of the Control Group in 1996, and was Head of Department 1999-2009. In 2009 he moved to Oxford as Professor of Control Engineering and Professorial Fellow at New College Oxford. He is now a Distinguished Professor at the University of Johannesburg, South Africa; an Extraordinary Professor at the University of Pretoria, South Africa; and an Emeritus Professor at the University of Oxford, UK. His research interests include applied and theoretical problems in control systems and engineering dynamics. He is a Life Fellow of the IEEE (1992), a Fellow of the IET (1994), a Fellow of the Royal Academy of Engineering (1997), a Fellow of the City and Guilds of London Institute, and a Fellow of the South African Academy of Engineering. He has received two South African Institute of Electrical Engineers Prizes and award certificates, the O. Hugo Schuck best paper award at the American Control Conference (with K. Glover), the 2015 IET Tustin Lecture, and the 2019 Honeywell International Medal from the Institute of Measurement and Control.

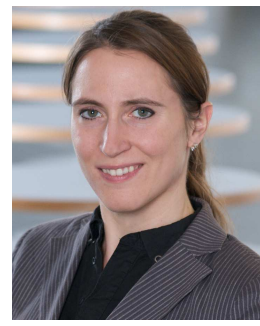

Sina Ober-Blöbaum received her Dipl. Math. in 2004 and her Ph.D. degree in applied mathematics in 2008 both from the University of Paderborn, Germany. She has been a Postdoctoral Scholar at California Institute of Technology, CA, USA, from 2008 to 2009. From 2009 to 2015 she has been a Junior Professor at the Department of Mathematics at the University of Paderborn. She is currently Associate Professor of control engineering at the Department of Engineering Science and a Tutorial Fellow in Engineering at Harris Manchester College, University of Oxford, Oxford, U.K. Her research focus lies in the development and analysis of structure-preserving simulation and optimal control methods for mechanical, electrical and hybrid systems, with a wide range of application areas including astrodynamics, drive technology and robotics.

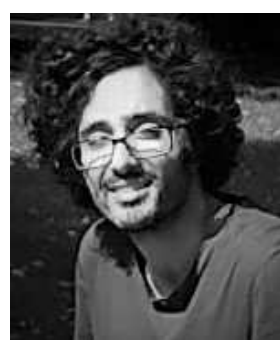

Farhang Haddad Farshi received his B.Sc. degree from the University of Tabriz in 2008, and M.Sc. from King's College London in 2012. He has been a research assistant at the Freiburg Institute for Advanced Studies from 2013 to 2014. He received his $\mathrm{PhD}$ degree in theoretical physics from Imperial College London in 2017. He is currently a post-doc researcher at the Department of Engineering Science at the University of Oxford. 